\title{
Enhancing model-based feedback perimeter control with data-driven online adaptive optimization
}

\author{
Anastasios Kouvelas, Mohammadreza Saeedmanesh, Nikolas Geroliminis* \\ Urban Transport Systems Laboratory \\ School of Architecture, Civil and Environmental Engineering \\ École Polytechnique Fédérale de Lausanne (EPFL) \\ GC C2 390, Station 18, CH-1015 Lausanne, Switzerland
}

\begin{abstract}
Most feedback perimeter control approaches that are based on the Macroscopic Fundamental Diagram (MFD) and are tested in detailed network structures restrict inflow from the external boundary of the network. Although such a measure is beneficial for the network performance, it creates virtual queues that do not interact with the rest of the traffic and assumes small unrestricted flow (i.e. almost zero disturbance). In reality, these queues can have a negative impact to traffic conditions upstream of the protected network which is not modelled. In this work an adaptive optimization scheme for perimeter control of heterogeneous transportation networks is developed and the aforementioned boundary control limitation is dropped. A nonlinear model is introduced that describes the evolution of the multi-region system over time, assuming the existence of well-defined MFDs. Multiple linear approximations of the model (for different set-points) are used for designing optimal multivariable integral feedback regulators. Since the resulting regulators are derived from approximations of the nonlinear dynamics, they are further enhanced in realtime with online learning/adaptive optimization, according to performance measurements. An iterative data-driven technique is integrated with the model-based design and its objective is to optimize the gain matrices and setpoints of the multivariable perimeter controller based on real-time observations.
\end{abstract}

\footnotetext{
${ }^{*}$ Corresponding author. Tel.: +41-21-69-32481; Fax: +41-21-69-35060.

Email addresses: tasos.kouvelas@epfl.ch (Anastasios Kouvelas), mohammadreza.saeedmanesh@epfl.ch (Mohammadreza Saeedmanesh), nikolas.geroliminis@epfl.ch (Nikolas Geroliminis)
}

Preprint submitted to Transportation Research Part B

October 4, 2016

(C) 2016. This manuscript version is made available under the Elsevier user license http://www.elsevier.com/open-access/userlicense/1.0/ 
The efficiency of the derived multi-boundary control scheme is tested in microsimulation for a large urban network with more than 1500 roads that is partitioned in multiple regions. The proposed control scheme is demonstrated to achieve a better distribution of congestion (by creating "artificial" interregional queues), thus preventing the network degradation and improving total delay and outflow.

Keywords: real-time urban perimeter control, macroscopic fundamental diagram, linear feedback regulators, online learning, adaptive optimization, adaptive fine-tuning (AFT).

\section{Introduction}

Real-time traffic management is deemed to be an efficient and cost effective way to ameliorate traffic conditions and prevent gridlock phenomena in cities. Although many methodologies have been developed for real-time signal control over the last decades (see e.g. Papageorgiou et al. (2003) for a good review), the design of efficient control strategies for heterogeneous large-scale urban networks that can deal with oversaturated conditions (where queues spill back to upstream links) remains a significant challenge. Local adaptive strategies that are widely used around the world are based on heuristic optimization techniques and are not efficient when the network faces congestion propagation phenomena and queue spillbacks. Other traffic responsive strategies (Gartner, 1983: Mirchandani and Head, 1998) use complex optimization methods, which make their online application to large-scale urban networks difficult due to high computational requirements.

More recently, a practicable network-wide control strategy (TUC) has been developed (Diakaki et al., 2002, 2003) that tries to deal with oversaturated conditions by minimizing the variance of relative occupancies of the network links; this strategy has been tested in simulation but also in various field implementations (see e.g. Aboudolas et al. (2010); Kouvelas et al. (2011b)). Another conception, that has been recently proposed for regulating urban traffic and is based on a decentralized approach of the problem, is the max-pressure controller (Varaiya, 2013; Kouvelas et al., 2014). This distributed control law, which was originally applied to production processes and communication networks and has lately gained a lot of attention in traffic control, acts locally in coupled intersections and has been proven (under certain conditions) to stabilize the queues of the network. In the same direction, 
Muralidharan et al. (2015) studied the network stability under fixed-time control; this analysis provides useful insights about simple network traffic instances (i.e. the demand is assumed to be "feasible" - can be accommodated by the signals) and can potentially lead to analytical derivations of performance measures (i.e. total travel time, total delay). Note, however, that in the case of heterogeneous networks with multiple pockets of congestion and heavily directional demand flows such an analysis is not straightforward (due to the lack of spillback modelling and infeasible demand ${ }^{1}$ ) and this type of control (i.e. TUC, max-pressure) may not be optimal or the stabilization of the system in a reasonable time period might not be feasible.

An alternative approach for real-time network-wide control for heterogeneous urban networks that is steadily gaining momentum is the perimeter flow control, which adds an additional layer of a more aggregated approach for modelling and control. While a global optimization framework for all controllers in the city may sound impossible (due to both computational burden and model uncertainty and unpredictability), identifying some critical intersections and regulating them effectively can significantly alleviate the level of congestion (and even make more efficient the local strategies). The basic concept of such an approach is to partition the heterogeneous network into a small number of homogeneous regions and apply perimeter control to the inter-transferring flows along the boundaries between regions. The input flows to a region (which are also output flows for the neighbouring regions) can be controlled at the intersections located at the borders of the region, so as to maximize the total throughput of the system. Perimeter control (or gating) policies have been introduced for single-region homogeneous networks (Daganzo, 2007; Keyvan-Ekbatani et al., 2012) and multi-region heterogeneous networks (Geroliminis et al. (2013); Aboudolas and Geroliminis (2013) and many other works) using different control methodologies. The key modelling tool that is used by all the aforementioned strategies is the Macroscopic Fundamental Diagram (MFD), which provides a concave, low-scatter relationship between network vehicle accumulation (veh) or density (veh/km) and network production $(\mathrm{veh} \cdot \mathrm{km})$ or circulating flow $(\mathrm{veh} / \mathrm{h})$. The concept of a network MFD was firstly introduced in Godfrey (1969), but the empirical

\footnotetext{
${ }^{1}$ This is a demand where one can prove mathematically that no feasible control can prevent congestion. One pathway to deal with this is demand management strategies (e.g. congestion pricing), but if this is not the case one can try to manage congestion in an efficient way for the system.
} 
verification of its existence with dynamic features is quite recent (Geroliminis and Daganzo, 2008).

Evidently, the stability of the MFD shape faces two main challenges which are (a) the hysteresis phenomena that appear at the onset or offset of congestion (Buisson and Ladier, 2009; Geroliminis and Sun, 2011a; Gayah and Daganzo, 2011a; Saberi and Mahmassani, 2012), and (b) the heterogeneity of traffic in urban networks (Mazloumian et al., 2010; Geroliminis and Sun, 2011b; Knoop et al., 2012). Essentially, heterogeneous networks do not exhibit a well-defined MFD, especially in the congested regime. Partitioning such a network into homogeneous regions (i.e. areas with compact shape that have small variance of link densities) can result in well-defined MFD as shown in Ji and Geroliminis (2012). Nevertheless, the MFD concept constitutes a useful tool for designing control policies, as it provides aggregated relationships between macroscopic traffic variables and reduces the complexity of traffic flow dynamics (i.e., there is no need for tracking the state of each individual link of the network).

Despite the vast literature related to empirical observations, modelling and control with MFDs, there are still multiple challenges in this growing field of research. In this work, we address 3 main challenges related to modelling, control and applicability of methodologies in real situations. First, (a) we reformulate the system dynamics developed in previous works in a way that the derived controllers can be implemented with limited data from inductive loop detectors. Second, (b) in the experimental studies all the controlled queues are internal to the simulated network and interact and influence the rest of the traffic and (c) an online data-driven approach is utilized to optimize the controller parameters.

With respect to (a) previous works have developed and described nonlinear dynamics of MFD systems with multiple regions (see e.g. Ramezani et al. (2015) for a detailed description). Nevertheless, these equations include state variables for vehicle accumulations $n_{i j}$ (where $i$ is the current region of vehicles and $j$ the destination region) and proper information about OD demand $d_{i j}$. If $n_{i j}$ and $d_{i j}$ can be measured with a decent level of accuracy, then the model predictive control approach developed in these works can properly solve the problem. However, there are some difficulties in estimating these variables without vehicle trajectories (i.e. only by using loop detector data). Thus, our current work does not contribute per se in the modelling of MFD dynamics, but rather adjusts previous formulations in a way that is very useful for control purposes without knowledge of $n_{i j}$ states. Regarding contributions (b) 
and (c) the work of Aboudolas and Geroliminis (2013) (for multiple regions) and Keyvan-Ekbatani et al. (2012) (for single region) specify set points $\hat{n}_{i}$ for each region $i$, which are integrated in the control framework. The specification of $\hat{n}_{i}$ for monocentric networks with clear attractions of destinations is treated properly by Aboudolas and Geroliminis (2013), while heterogeneous networks with multiple regions of attraction would require a non-trivial choice of $\hat{n}_{i}$. Physically speaking, if a control approach can keep all regions below or close to the critical accumulation of each $\mathrm{MFD}-n_{i}$ that maximizes the regional outflow - then the problem is well resolved by previous works (see Aboudolas and Geroliminis (2013) for multiple regions and Keyvan-Ekbatani et al. (2012) for single region problems). A challenge that we address in this work is the case where keeping all regions at the desired $\hat{n}_{i}$ is not possible. For example, if heavily directional flows from the periphery of a network pass through a small region to enter the center, the set point of the small region should be smaller than the set point of the periphery. The fact that we do not control the external boundary of the network, makes such a consideration crucial, as keeping all regions uncongested or at the critical accumulation might not be feasible.

\subsection{State of the art of perimeter control}

There are some recent works (Geroliminis et al., 2013; Aboudolas and Geroliminis, 2013; Keyvan-Ekbatani et al., 2015) that deal with perimeter control for multi-region systems with MFD-based modelling. However, none of the above works deals with parameter uncertainties or short-term and long-term variations in the system dynamics, i.e. all model parameters are deterministic and the behaviour of the model does not change over time ${ }^{2}$ In Haddad et al. (2013); Ramezani et al. (2015) a model predictive control approach is proposed and a nonlinear MFD-based model is used to describe the dynamics of the system. Although the controller is tested for different errors in the MFDs and the demand profiles, perfect knowledge of the model parameters is assumed. In Aboudolas and Geroliminis (2013) a multivariable linear quadratic state feedback regulator is studied for perimeter control

\footnotetext{
${ }^{2}$ As a consequence, a defect of some field implementations is that they do not consider the adaptation of the system to external disturbances that can affect the dynamics (e.g. seasonal effects, changes in driving behaviour and/or infrastructure characteristics). In many cases the utilized models are calibrated once and are not re-calibrated in a regular basis.
} 
and two versions of the optimization problem are tested (with (LQI) and without (LQR) integral action). The LQI/LQR gain matrices are designed by linearising the nominal nonlinear traffic dynamics around a predefined set-point. Note that such nominal optimal control laws do no guarantee the robustness properties with respect to uncertainties. In this case study the inflow from the external boundary of the network is restricted by the regulator, creating virtual point queues that do not interact with traffic upstream of the protected regions. A more recent work (Haddad and Mirkin, 2016) utilizes the context of model reference adaptive control in order to improve the performance of feedback controllers under uncertainties. The derived controllers incorporate input delay and can deal with bounded external dependencies. Finally, a conventional pathway to address this problem is through robust control design and recently there have been some notable efforts in this direction (see e.g. Haddad and Shraiber (2014); Haddad (2015)). These approaches can effectively deal with parameter uncertainties, but, on the other hand, the control actions may in some cases be quite conservative (if many stochastic scenarios are generated). Finally, the studies in Gayah and Daganzo (2011a); Haddad and Geroliminis (2012); Gayah et al. (2014) reveal some fruitful insights about the stability and robustness of MFD-based systems under different adaptive signal approaches for systems with simplified dynamics and topologies.

In this work we revisit and readjust previous formulations of MFD dynamics of multiple regions (Ramezani et al., 2015), to derive a generic MFD-model that can deal with limited data and simpler state description. Multiple linear approximations of the model (for different set-points) are exploited to derive optimal multivariable proportional integral (PI) feedback regulators. These regulators are applied to the multi-region network and provide an initial set of observations of the system. Then, the gain matrices and set-points of the PI controller are updated in real-time by an adaptive optimization algorithm based on performance measurements. The set of observations that is obtained by the linearised model together with the real measurements are inputs to the algorithm and provide some initial samples for the online learning procedure. Microsimulation experiments demonstrate the versatility and real-time applicability of the approach.

A novelty of this case study is that the queues created due to control actions are inside the microsimulaton model. Note, that all the perimeter or gating control strategies that utilise MFD modelling and have been tested in microsimulation environment (e.g. Keyvan-Ekbatani et al. (2012); Aboudolas 
and Geroliminis (2013); Keyvan-Ekbatani et al. (2015)), apply control in the external boundary of the network and as a consequence queued vehicles create point queues that do not interact or constrain other movements. This is similar to applying ramp-metering control on a motorway and assume infinite storage capacity on the on-ramps, which might be problematic if queues spillback in the urban network. In reality, movements outside the protected zones might be influenced by these queues and this is not modelled in microsimulation. In this work, the protected zones/boundaries are internal to the network and interactions are taken into consideration. This approach significantly challenges the performance of the feedback regulator, as (a) the disturbance in the system due to uncontrolled inflow is higher compared to systems that restrict the flow at the external boundaries of the network and (b) queues at the boundaries between regions can affect other movements (e.g. perpendicular to the boundary) due to spillbacks.

Another issue that is investigated is the optimal selection of MFD setpoints for antagonistic regions. The specification of set-points for monocentric networks (Keyvan-Ekbatani et al., 2015) with well-defined destination attractions is straightforward, as the objective is to operate the protected regions around the critical accumulation that maximizes flow. In this case study, we show that the aforementioned strategies might not succeed in bringing the system to the desired states. Nevertheless, heterogeneous networks with multiple regions of attraction would require a non-trivial choice of set-points (which are related to the level of congestion in each region). Physically speaking, if a control approach can keep all regions below or close to the critical accumulation of each MFD, then the problem is well resolved (see e.g. Aboudolas and Geroliminis (2013)). However, in the case of excessive demand where not all regions can be uncongested, one region could be favoured against another one (through the set-points selection) if this is beneficiary for the overall system. In cases of high demand/supply ratios that congestion can provably not be avoided the objective is to optimally distribute the congestion across the regions. One may select to "protect" a region that attracts many trips (master) and let another region be congested (slave) if it is not crucial for the system delay, in order to maximize system throughput. A challenge, which is investigated here, is the optimal choice of set-points that can lead heterogeneous systems in desired states with minimum congestion by utilizing the master-slave concept. While model predictive approaches (see e.g. Ramezani et al. (2015)) can identify close-to-optimal control policies that minimize system-wide delays, unreliable predictions might harden the 
procedure.

In this work we try to overcome all the aforementioned difficulties by combining model-based optimal control design with data-driven online tuning

of the regulator. An automatic fine-tuning algorithm (building on Kouvelas et al. (2011a)) is integrated to identify optimal values for the set-points (and gains) in an online adaptive mode. AFT (Adaptive Fine-Tuning) is an iterative algorithm that receives a scalar performance index (e.g. total delay) for different sets of controller parameters (gain matrices ans set-points) and tries to learn the nonlinear mapping between these parameters and the regulator performance. In each iteration AFT uses this knowledge in order to update the values of the parameters aiming at better performance. The control inputs consist of the ratios of inter-transferring flows between neighbouring regions and the actuators correspond to the traffic lights of these areas (e.g. inter-regional boundaries). The overall control scheme is tested in microsimulation for the urban network of Barcelona, Spain, which includes more than 600 intersections and the impact of the applied perimeter control is evaluated via the corresponding MFDs and other performance measures. The fixed-time policy of the city is compared to the final controller that is obtained after the convergence of AFT.

The remainder of the paper is organized as follows: Section 2 presents the modelling of aggregated dynamics for an urban network partitioned in $N$ regions. In Section 3, a linear optimal control methodology is described that can be applied to different linear approximations of the model. The designed control is enhanced in real-time by a data-driven adaptive optimization algorithm, which is described in details in Section 4. Finally, the integrated control scheme is applied to the network of Barcelona in microsimulation and the obtained results are presented in Section 5. The main conclusions are summarized in Section 6 .

\section{Modelling of a multi-region MFD-based system}

Consider an urban network partitioned in $N$ homogeneous regions (Figure 1(a). The index $i \in \mathcal{N}=\{1,2, \ldots, N\}$ denotes the region of the system and $n_{i}(t)$ the total accumulation (number of vehicles) in region $i$ at a given time $t$. Let $\mathcal{N}_{i}$ be the set of all regions that are directly reachable from the borders of region $i$ (i.e. adjacent regions to region $i$ ) and $q_{i \text {,in }}(t), q_{i, \text { out }}(t)$ the inflow and outflow of region $i$ at time $t$, respectively. Also, let $d_{i}(t)$ denote the total uncontrolled traffic demand (disturbance) in region $i$ at time $t$. Note 


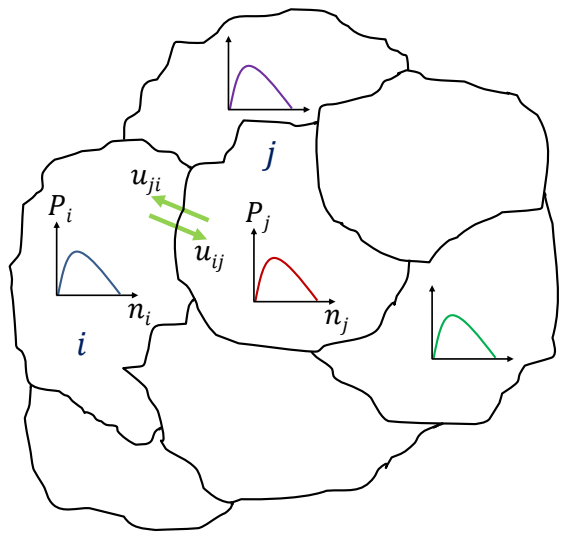

(a)

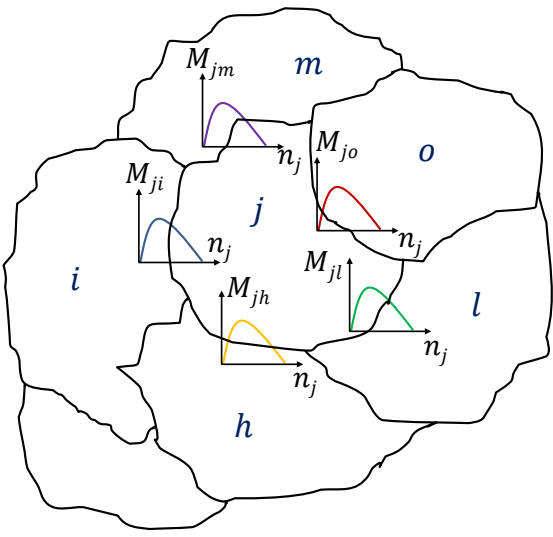

(b)

Figure 1: A network modelled as a multi-region MFDs system: (b) production MFDs; (b) sending flows MFDs.

that $d_{i}(t)$ includes both the internal generated demand (vehicles entering the network from on-street and off-street parking areas) and the external uncontrollable inflows. The conservation equation for each region $i$ of the system reads (as in most of the aforementioned papers dealing with MFD and perimeter control)

$$
\frac{d n_{i}(t)}{d t}=q_{i, \text { in }}(t)-q_{i, \text { out }}(t)+d_{i}(t)
$$

For every region $i$ it is assumed that there exists a production MFD relating the accumulation $n_{i}(t)$ to the total production $P_{i}\left(n_{i}(t)\right)$ and describes the performance of the sub-system in an aggregated way. This MFD can be easily estimated using measurements from loop detectors and/or GPS trajectories. The total outflow $O_{i}$ of region $i$ (number of vehicles exiting the region per unit time, either because they finished their trip or because they move to a neighbouring region) can be estimated by $O_{i}\left(n_{i}(t)\right)=P_{i}\left(n_{i}(t)\right) / L_{i}$, where $L_{i}$ is the average trip length for region $i$, which is assumed to be independent of time and destination, internal or external, in $i$. Furthermore, let $M_{i j}\left(n_{i}(t)\right),(i \neq j)$ denote the sending flow from region $i$ to region $j$ at time $t$. This variable can also be related to the accumulation $n_{i}(t)$ by using an MFD (as demonstrated later) and estimated by using the measurements of all the detectors located in the borders between regions $i$ and $j$ (Figure 1(b)). Finally, $M_{i i}\left(n_{i}(t)\right.$ ) denotes the internal trip completion rate in region $i$ (vehicles finishing their trip inside 
the region) and is given by

$$
M_{i i}\left(n_{i}(t)\right)=O_{i}\left(n_{i}(t)\right)-\sum_{j \in \mathcal{N}_{i}} M_{i j}\left(n_{i}(t)\right)
$$

\subsection{Outflow model discrepancy}

The model $O_{i}\left(n_{i}(t)\right)=P_{i}\left(n_{i}(t)\right) / L_{i}$ mentioned above approximates the outflow of a region under certain assumptions and has been used in various works in the literature. It is an adequate model for control design as it derives simplified system dynamics without delays, i.e., it considers that the outflow is always given by the ratio of production over trip length (and trip length is assumed constant). While there are empirical verifications about the validity of this model with aggregated data (e.g. Geroliminis and Daganzo (2008)) it should not be considered a universal law. Strong fluctuations in the demand that create fast evolving transient states, spatial heterogeneity of congestion or route choice effects can influence the trip length distribution of vehicles in a region at a specific time and $P_{i}$ over $L_{i}$ approximation of outflow might experience some errors. While we consider this a valid assumption for a range of cases, further research both in the theoretical and empirical side would be useful to investigate this limitation, but this is out of the scope of the present work. Here, it is used only for calculating $M_{i i}\left(n_{i}(t)\right)$ for every region $i$, as it is not straightforward to measure this quantity in reality without probe vehicle data.

Note also, that the transfer flow can be lower than the sending flow $M_{i j}$, as it can be restricted by control actions $u_{i j} \in[0,1]$; the actual transfer flow is equal to $u_{i j} M_{i j}$. Nevertheless, equation (2) should not include any control variable $u_{i j}$, as this would mean that if the sending flow is restricted, then the internal trip completion rate $M_{i i}$ could increase (consider the extreme case where $u_{i j}=0, \forall j \in \mathcal{N}_{i}$; then still $M_{i i}<O_{i}(n i(t))$ should hold in equation (2)). Previous works with model predictive control Geroliminis et al., 2013, Ramezani et al. 2015) estimate the flows $M_{i j}$ by utilizing more detailed description of the system states, i.e. $M_{i j}\left(n_{i j}(t), n_{i}(t)\right)=\min \left\{n_{i j}(t) / n_{i}(t) \cdot O_{i}\left(n_{i}(t)\right), C_{j}\left(n_{j}(t)\right)\right\}$, where $n_{i j}$ describes the number of vehicles in region $i$ with $j$ as the next destination and $C_{j}\left(n_{j}(t)\right)$ the receiving capacity of region $j$, which is a piecewise function of $n_{j}(t)$ with two pieces, one constant value and a decreasing curve. As it is difficult to estimate the variables $n_{i j}$ without probe vehicle information and the current work mainly utilizes loop detector data to estimate the system states, the functions of sending flows $M_{i j}$ are directly estimated by the measurements of the fixed-time control scenario. 
The description of system states that embeds constraints in the outflow based on the receiving region has been discussed in previous publications (first in Geroliminis and Daganzo (2007), also in Haddad et al. (2013); Ramezani et al. (2015); Knoop and Hoogendoorn (2014)). Indeed, the most comprehensive dynamic equations are the ones presented in Ramezani et al. (2015), where the sending flow $M_{i j}$ is a function of $n_{i j}, n_{i}$, but also $n_{j}$ and the spatial heterogeneity of link density in region $i$. It is clear that our approximation simplifies the dynamics of the network, but the feedback control design is able to treat this assumption properly. Note also (as it has been discussed in the aforementioned paper), that the receiving (or boundary) capacity constraint can be omitted during the optimization process without introducing stability issues, as (a) boundary capacity decreases for accumulations much larger than the critical accumulation (see Geroliminis and Daganzo (2007) - we have also estimated the boundary capacity of the network of Barcelona and this conclusion is valid for our experiments), (b) the control inputs will not allow the system to get close to gridlock, and (c) feedback can contribute in these effects (with some delay of course). The model still provides a decent description of system dynamics even under adaptive control conditions.

\subsection{Aggregated system dynamics}

The inflow to region $i$ is the summation of the transferring flows from all its neighbouring regions and is given by

$$
q_{i, \text { in }}(t)=\sum_{j \in \mathcal{N}_{i}} u_{j i}(t) M_{j i}\left(n_{j}(t)\right)
$$

where the control variables $u_{j i}(t), \forall j \in \mathcal{N}_{i}, i \in \mathcal{N}$ denote the fraction of the flow that is allowed to transfer from region $j$ to region $i$ at time $t$, to be calculated by the perimeter controller. Equivalently, the outflow of region $i$ is the summation of the transferring flows to all its neighbouring regions plus the trip completion rate in region $i$ and is given by

$$
q_{i, \text { out }}(t)=M_{i i}\left(n_{i}(t)\right)+\sum_{j \in \mathcal{N}_{i}} u_{i j}(t) M_{i j}\left(n_{i}(t)\right)
$$

The values of the control variables $u_{i j}$ are constrained by physical or operational constraints as follows

$$
0<u_{i j, \min } \leq u_{i j}(t) \leq u_{i j, \max }<1, \quad \forall i \in \mathcal{N}, j \in \mathcal{N}_{i}
$$


where $u_{i j, \min }, u_{i j, \max }$ are the minimum and maximum permissible transferring rates of flows, respectively. Also, each region $i$ has a maximum accumulation $n_{i, \max }$, i.e. $0 \leq n_{i}(t) \leq n_{i, \max }, \forall i \in \mathcal{N}$, and if $n_{i}(t)=n_{i, \max }$ then the region reaches gridlock and all the inflows along the periphery are restricted. Invoking (3)-(4) and (1) the following non-linear state equation is obtained

$$
\frac{d n_{i}(t)}{d t}=\sum_{j \in \mathcal{N}_{i}} u_{j i}(t) M_{j i}\left(n_{j}(t)\right)-M_{i i}\left(n_{i}(t)\right)-\sum_{j \in \mathcal{N}_{i}} u_{i j}(t) M_{i j}\left(n_{i}(t)\right)+d_{i}(t)
$$

Note that $(6)$ is a more accurate representation of system dynamics compared to Aboudolas and Geroliminis (2013), i.e. the outflows of the regions are controlled since the exiting (sending) flows from region $i, M_{i j}$, are multiplied with the control variables $u_{i j}$, which is necessary as there are some uncontrolled boundaries that create larger disturbance to the system.

This nonlinear model may be linearized around some set-point $\left(\hat{n}_{i}, \hat{n}_{j}, \hat{u}_{i j}\right.$, $\left.\hat{u}_{j i}, \hat{d}_{i}\right), j \in \mathcal{N}_{i}$. The selection of $\hat{n}_{i}$ is closely related to the existence of MFDs $O_{i}\left(n_{i}(t)\right), M_{i j}\left(n_{i}(t)\right)$, which are approximated by third degree polynomial functions of $n_{i}(t)$ and provide a critical accumulation at which the performance of the region is optimized. The desired set-point should satisfy the steady-state version of (6) which reads

$$
0=\sum_{j \in \mathcal{N}_{i}} \hat{u}_{j i} M_{j i}\left(\hat{n}_{j}\right)-M_{i i}\left(\hat{n}_{i}\right)-\sum_{j \in \mathcal{N}_{i}} \hat{u}_{i j} M_{i j}\left(\hat{n}_{i}\right)+\hat{d}_{i}
$$

The solution of the linear system of equations (i.e. steady-state for every region $i$ ) for given $\hat{n}_{i}, \hat{d}_{i}$, provides the nominal control actions $\hat{u}_{i j}, \forall i \in \mathcal{N}_{i}, j \in \mathcal{N}$ that stabilize the system around the equilibrium point. Note that this system of linear equations may have multiple feasible solutions (depending on the layout of the network). By denoting $\Delta x=x-\hat{x}$ analogously for all variables the linearization of $(6)$ around the selected set-point yields

$$
\begin{aligned}
\Delta \dot{n}_{i}(t)= & \sum_{j \in \mathcal{N}_{i}} \Delta u_{j i}(t) M_{j i}\left(\hat{n}_{j}(t)\right)+\sum_{j \in \mathcal{N}_{i}} \hat{u}_{j i}(t) \Delta n_{j}(t) M_{j i}^{\prime}\left(\hat{n}_{j}(t)\right) \\
& -\Delta n_{i}(t) M_{i i}^{\prime}\left(\hat{n}_{i}(t)\right)-\sum_{j \in \mathcal{N}_{i}} \Delta u_{i j}(t) M_{i j}\left(\hat{n}_{i}(t)\right) \\
& -\sum_{j \in \mathcal{N}_{i}} \hat{u}_{i j}(t) \Delta n_{i}(t)(t) M_{i j}^{\prime}\left(\hat{n}_{i}(t)\right)+\Delta d_{i}(t)
\end{aligned}
$$


By applying the linear equation (8) to an urban network partitioned in $N$ regions, the following state equation is obtained (in compact vector form) that describes the evolution of the system in time

$$
\Delta \dot{\mathbf{n}}(t)=\overline{\mathbf{A}} \Delta \mathbf{n}+\overline{\mathbf{B}} \Delta \mathbf{u}+\overline{\mathbf{C}} \Delta \mathbf{d}
$$

where $\Delta \mathbf{n} \in \mathbb{R}^{N}$ is the state deviations vector $\Delta n_{i}=n_{i}-\hat{n}_{i}, \forall i \in \mathcal{N}$; $\Delta \mathbf{u} \in \mathbb{R}^{M}$ is the control deviations vector $\Delta u_{i j}=u_{i j}-\hat{u}_{i j}, \forall i \in \mathcal{N}, j \in \mathcal{N}_{i}$; $\Delta \mathbf{d} \in \mathbb{R}^{N}$ is the demand deviations vector $\Delta d_{i}=d_{i}-\hat{d}_{i}, \forall i \in \mathcal{N} ; \overline{\mathbf{A}} \in \mathbb{R}^{N \times N}$, $\overline{\mathbf{B}} \in \mathbb{R}^{N \times M}$ are the appropriate state and control matrices, that are derived by application of (8), $\forall i \in \mathcal{N}, j \in \mathcal{N}_{i} ; \overline{\mathbf{C}}=\mathbf{I}_{N \times N}$ is the identity matrix.

The continuous time linear state system (9) of the multi-region system may be directly translated in discrete time (with sample time $T$ ) by use of standard formulas (e.g. zero-order hold method (Brown and Hwang, 1997)). The resulting discrete time system in vector form reads

$$
\Delta \mathbf{n}(k+1)=\mathbf{A} \Delta \mathbf{n}(k)+\mathbf{B} \Delta \mathbf{u}(k)+\Delta \mathbf{d}(k)
$$

where $k=0,1, \ldots, K-1$ is the discrete time index and $\mathbf{A} \in \mathbb{R}^{N \times N}, \mathbf{B} \in \mathbb{R}^{N \times M}$ are the corresponding discrete time state and control matrices.

\section{Model-based design of multivariable PI feedback regulators}

The discrete time linear system (10) approximates the original non-linear system around the set-point and can be used for application of efficient methodologies from linear MIMO (multiple-input-multiple-output) optimal control theory. The approach of Linear-Quadratic-Integral (LQI) control is employed here.

\subsection{The Linear-Quadratic-Integral methodology}

In this approach the state of the system 10 is augmented by additional state variables that integrate the error signal $\Delta \mathbf{n}$, which is then used as a feedback term to provide zero steady-state error. The new state variables are given by

$$
\Delta \mathbf{y}(k+1)=\Delta \mathbf{y}(k)+\mathbf{C} \Delta \mathbf{n}(k)
$$

where $\Delta \mathbf{y} \in \mathbb{R}^{Z}$ is the integral vector and $\mathbf{C} \in \mathbb{R}^{Z \times N}$ is typically a binary matrix (i.e. its entries are 0 or 1 ), such that $Z$ components (or linear combinations of components) of the system state are integrated in (10). Note that 
$N+Z \leq M$ must hold in order for the system to be fully controllable. The augmented discrete time system (10)-(11) can be written in compact form as

$$
\Delta \tilde{\mathbf{n}}(k+1)=\tilde{\mathbf{A}} \Delta \tilde{\mathbf{n}}(k)+\tilde{\mathbf{B}} \Delta \mathbf{u}(k)+\tilde{\mathbf{C}} \Delta \mathbf{d}(k)
$$

where $\tilde{\mathbf{n}}(k)=\left[\begin{array}{ll}\Delta \mathbf{n}(k) & \Delta \mathbf{y}(k)\end{array}\right]^{\top}$ is the augmented state vector and $\tilde{\mathbf{A}}, \tilde{\mathbf{B}}, \tilde{\mathbf{C}}$ are the augmented state, control, and demand matrices, respectively, which are given by

$$
\tilde{\mathbf{A}}=\left[\begin{array}{ll}
\mathbf{A} & \mathbf{0} \\
\mathbf{C} & \mathbf{I}
\end{array}\right], \quad \tilde{\mathbf{B}}=\left[\begin{array}{l}
\mathbf{B} \\
\mathbf{0}
\end{array}\right], \quad \tilde{\mathbf{C}}=\left[\begin{array}{l}
\overline{\mathbf{C}} \\
\mathbf{0}
\end{array}\right]
$$

Finally, for formulating the LQI optimal control problem the following quadratic objective criterion is defined

$$
\begin{array}{rl}
\min _{\mathbf{u}} & \mathcal{J}(\mathbf{u})= \\
& \frac{1}{2} \sum_{k=0}^{K}\left(\Delta \mathbf{n}^{\top}(k) \mathbf{Q} \Delta \mathbf{n}(k)+\Delta \mathbf{u}^{\top}(k) \mathbf{R} \Delta \mathbf{u}(k)+\Delta \mathbf{y}^{\boldsymbol{\top}}(k) \mathbf{S} \Delta \mathbf{y}(k)\right)
\end{array}
$$

where $\mathbf{Q} \in \mathbb{R}^{N \times N}, \mathbf{R} \in \mathbb{R}^{M \times M}$ and $\mathbf{S} \in \mathbb{R}^{Z \times Z}$ are user-defined diagonal weighting matrices that can influence the magnitude of each term of the objective criterion and are usually defined via trial-and-error. The optimal closed-loop solution of (14) subject to $(12)$ for an infinite time horizon (i.e. $K=\infty$ ), and assuming $\Delta \mathbf{d}(k)=\mathbf{0}$, leads to the following multivariable PI feedback regulator (see Papageorgiou et al. (2012) for details)

$$
\mathbf{u}(k)=\mathbf{u}(k-1)-\mathbf{K}_{\mathrm{P}}[\mathbf{n}(k)-\mathbf{n}(k-1)]-\mathbf{K}_{\mathrm{I}}[\mathbf{n}(k)-\hat{\mathbf{n}}]
$$

where $\mathbf{K}_{\mathrm{P}}, \mathbf{K}_{\mathrm{I}} \in \mathbb{R}^{M \times N}$ are the proportional and integral gains of the regulator, which are computed by the solution of the corresponding discrete-time Riccati equation and depend only on the matrices $\tilde{\mathbf{A}}, \tilde{\mathbf{B}}, \mathbf{Q}, \mathbf{R}$ and $\mathbf{S}$ defined above. In case that future demand flow predictions are available (i.e. $\Delta \mathbf{d}(k) \neq \mathbf{0}$, in (12)), simple feedforward control techniques can be used to integrate the disturbance predictions to the problem solution (Papageorgiou et al., 2012). It should be noted that the number of control variables $M$ depends on the network partition and the sets $\mathcal{N}_{i}, i \in \mathcal{N}$, yet for any arbitrary formation of the $N$-region system $N \leq M$ always holds. Finally, it should be emphasized that a well-known property of the PI regulator (15) is that it provides zero steady-state error (due to the existence of the integral term), i.e. $\mathbf{n}(k)=\hat{\mathbf{n}}$ under stationary conditions. Nevertheless, the traffic conditions are rarely stationary and the controller in (15) can be further improved through the automatic fine tuning method presented later. 


\subsection{Implementation aspects}

The state feedback regulator 15 is activated in real-time at each control interval $T$ and only within specific time windows based on the current accumulations $\mathbf{n}(k)$ (i.e. by use of two thresholds $n_{i \text {,start }}$ and $n_{i, \text { stop }}{ }^{3}$ and real-time measurements). The required real-time information of the vehicle accumulations $\mathbf{n}(k)$ can be directly estimated via loop detector time-occupancy measurements. Furthermore, in cases where only sparse measurements are available, different approaches to estimate MFD related state variables with real data are described in Leclercq et al. (2014), Ortigosa et al. (2014) and Ampountolas and Kouvelas (2015).

Equation (15) calculates the fraction of flows $\mathbf{u}(k)$ to be allowed to transfer between neighbouring regions. It should be emphasized that the solution of LQI methodology does not take into consideration any type of constraints for the state or control variables. In case the ordered values $u_{i j}(k)$ violate the constraints (5) they should be adjusted to become feasible, i.e. truncated to $\left[u_{i j, \min }, u_{i j, \max }\right]$. Moreover, the values of $\mathbf{u}(k-1)$ used on the right-hand side of (15), should be the bounded values of the previous time step (i.e. after the application of the constraints) in order to avoid possible wind-up phenomena in the PI regulator. The obtained $u_{i j}(k)$ values are then used to derive the green time durations for the stages of the signalized intersections located at the inter-regional boundaries. For a given pair of sending and receiving regions, $i, j$, respectively, the ordered transferring flow by the controller is $u_{i j}(k) M_{i j}\left(n_{i}(k)\right)$ vehicles per time unit. This flow is distributed to the corresponding intersections proportionally to the saturation flows of the controlled links (i.e., typically, links with more lanes are anticipated to accommodate more flow). To this end, every link $z$ is required to transfer $u_{i j}(k) M_{i j}\left(n_{i}(k)\right) S_{z} / S_{i j}$ flow, where $S_{z}$ is the saturation flow of the link and $S_{i j}$ the summation of all saturation flows related to the $i \rightarrow j$ movement. The green stage duration $g_{z}$ is given by $g_{z}(k)=u_{i j}(k) M_{i j}\left(n_{i}(k)\right) C_{i j} / S_{i j}$, where $C_{i j}$ defines the cycle time; without loss of generality $C_{i j}$ is assumed to be equal for all intersections included in the $i \rightarrow j$ movement (i.e. the equation can be readily modified if this assumption does not hold). Note that the real transferring flows may be different than the ordered ones for different reasons (e.g. low demand, spillback from downstream links); however, the regulator is

\footnotetext{
${ }^{3}$ In practical applications usually $n_{i, \text { stop }}<n_{i, \text { start }}$ is selected in order to avoid frequent activations/deactivations of the controller.
} 
robust to these occurrences due to its feedback structure (i.e. the differences will be integrated into the measurements of the following control cycles).

The structure of the controller (15) is similar to the one used in Aboudolas and Geroliminis (2013) although derived by a more accurate model. Moreover, here there are no control variables at the external borders of the network, but only at the inter-regional borders. As a consequence, there are no vehicles kept outside of the network in order to protect the congestion of the regions (which is also the case for most of the gated intersections in Keyvan-Ekbatani et al. (2012)) and all the queues created by the controller are internal to the network and thus affecting other movements. More importantly, in the current work the gain matrices $\mathbf{K}_{\mathrm{P}}, \mathbf{K}_{\mathrm{I}}$ and set-points $\hat{\mathbf{n}}$ of the controller are optimized in real-time by a learning/adaptive algorithm based on real performance measurements, ensuing a more realistic set-up of the problem. The closed-loop adaptive optimization scheme that constantly updates the parameters of the controller is presented in the next section. Our analysis shows that due to the additional complexities related to uncontrolled external boundaries, previous PI-type strategies are not capable to perform at the desired states (see Section 5.4 without a proper framework of adaptation, which is described in the next section.

\section{Adaptive optimization algorithm description}

The parameters $\mathbf{K}_{\mathrm{P}}, \mathbf{K}_{\mathrm{I}}$, $\hat{\mathbf{n}}$ of the regulator 15 are updated in real-time based on measurements of an objective function, so as to optimize the performance of the controller. The Adaptive Fine-Tuning (AFT) algorithm is used for that purpose. AFT is a recently developed algorithm (see Kosmatopoulos and Kouvelas (2009); Kouvelas et al. (2011a) for details) for tuning the parameters (in the specific case the gain matrices and set-points) of controller in an optimal way. It is an iterative algorithm that is based on machine learning techniques and adaptive optimization principles and adjusts the control gains and set-points to uncertainties and variations of the process under control. The working principle of the integrated closed-loop system (PI regulator and AFT) is presented in Figure 2 and may be summarized as follows:

- The $N$-region MFDs system is controlled in real-time by the multivariable PI regulator (15) which includes a number of tunable parameters $\boldsymbol{\theta} \triangleq \operatorname{vec}\left(\mathbf{K}_{\mathrm{P}}, \mathbf{K}_{\mathrm{I}}, \hat{\mathbf{n}}\right)$, where $\boldsymbol{\theta}$ is a vector with size $2 \times(M \times N)+N$ and its elements are the entries of $\mathbf{K}_{\mathrm{P}}, \mathbf{K}_{\mathrm{I}}, \hat{\mathbf{n}}$ taken column-wise. 
- At the end of appropriately defined periods $T_{\mathrm{c}}$ (e.g. at the end of each day), AFT algorithm receives the value of the real (measured) performance index $J$ (e.g. total delay of the system), as well as the values of the most significant measurable external disturbances $\mathbf{x}$ (e.g. aggregated demand). Note that the scalar performance index $J(\boldsymbol{\theta}, \mathbf{x})$ is a (generally unknown) function of the external factors $\mathbf{x}$ and the tunable parameters $\boldsymbol{\theta}$.

- Using the measured quantities (the samples of which increase iteration by iteration), AFT calculates new values for the tunable parameters to be applied at the next period (e.g. the next day) in an attempt to improve the system performance.

- This (iterative) procedure is continued over many periods (e.g. days) until the algorithm converges and an optimal performance is reached; then, AFT algorithm may remain active for continuous adaptation or can be switched off and re-activated at a later stage.

The main component of the employed algorithm is a universal approximator $\hat{J}(\boldsymbol{\theta}, \mathbf{x})$ (e.g., a polynomial-like approximator or a neural network) that is used in order to obtain an approximation of the nonlinear mapping $J(\boldsymbol{\theta}, \mathbf{x})$, based on all previous samples. At each iteration $k_{\mathrm{c}}$, the algorithm uses all the collected data for the sets of parameters applied at iterations $1,2, \ldots, k_{\mathrm{c}}$ and performs the steps described in Algorithm 1 in order to determine the new set of parameters for the next period (e.g. next day). A shortcoming of AFT approach (as for most data-driven learning algorithms) is that the first iterations might create controllers with poor performance and unacceptable behaviour (as shown later), due to the lack of information. While the performance of the designed controller will improve after some iterations, real life implementation would be problematic as the first trials might create very congested conditions in the system. In our approach, we perform the first iterations based on different mulrivariable PI regulators, that are obtained by solving the Riccati equation (see problem formulation (12) and (14) for different desired states. This allows for good quality initial solutions that overcome the discrepancies of first AFT iterations and provide data samples for the learning procedure. The combination of the offline model-based control design presented in Section 3.1 and the online data-driven adaptation with AFT provides a robust approach for dealing with the perimeter control problem, as it will be demonstrated in the next section. 


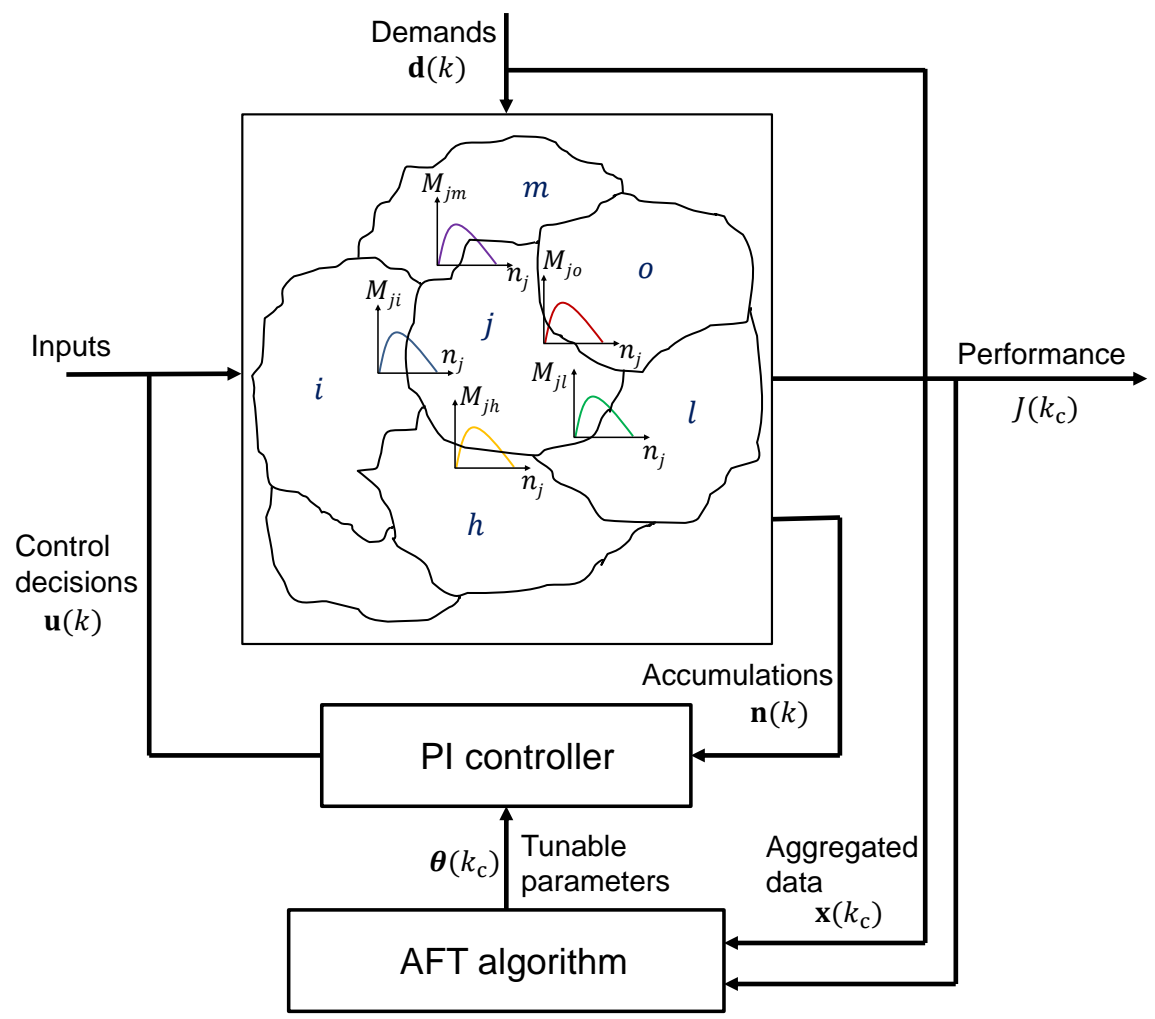

Figure 2: The integrated closed-loop adaptive system (multivariable PI regulator and AFT algorithm).

\section{Application of the perimeter control scheme to a large network}

The efficiency of the adaptive flow control scheme described in the previous sections is tested in microsimulation experiments. The Aimsun microscopic environment (Version 8.0.8) is used and the real-time implementation of the control scheme is replicated through the simulator API. Only loop detector data is utilized to estimate the state of the system, highlighting the feasibility and applicability of the developed framework in a real life conditions.

\subsection{Network description}

The urban network of Barcelona, Spain is used as the test site (see Figure 3(a) for a representation of the studied area on the map). The network covers an area of 12 square kilometers with about 600 intersections and 1500 links of various lengths and is modelled and calibrated in Aimsun (Figure 3(c)] . 
Algorithm 1: AFT algorithm for iteration $k_{\mathrm{c}}$ (originally proposed in Kouvelas et al. (2011a)).

Data: $\alpha_{0}, L_{\mathrm{g}, \max }, \boldsymbol{\theta}_{\min }, \boldsymbol{\theta}_{\max }$, all available measurements of vectors $\boldsymbol{\theta}, \mathbf{x}$ and scalar $J$ up to iteration $k_{\mathrm{c}}$.

Result: Calculate the new set of tunable parameters $\boldsymbol{\theta}\left(k_{\mathrm{c}}+1\right)$ to be applied at the next period $k_{\mathrm{c}}+1$.

1: Set $L_{\mathrm{g}}=\min \left\{2\left(k_{\mathrm{c}}-1\right), L_{\mathrm{g}, \max }\right\}$.

2: Produce a new polynomial approximator with $L_{\mathrm{g}}$ regressor terms, which has the following structure

$$
\hat{J}^{\left(k_{\mathrm{c}}\right)}(\boldsymbol{\theta}, \mathbf{x})=\boldsymbol{\vartheta}^{\top}\left(k_{\mathrm{c}}\right) \boldsymbol{\phi}^{\left(k_{\mathrm{c}}\right)}(\boldsymbol{\theta}, \mathbf{x})
$$

where $\boldsymbol{\vartheta}\left(k_{\mathrm{c}}\right) \in \mathbb{R}^{L_{\mathrm{g}}}$ are the weights of the approximator for iteration $k_{\mathrm{c}}$ (equivalent to the synaptic connections in neural networks) and $\phi^{\left(k_{c}\right)}(\boldsymbol{\theta}, \mathbf{x})$ is a vector with $L_{\mathrm{g}}$ sigmoidal functions of polynomials constructed using the elements of vectors $\boldsymbol{\theta}, \mathbf{x}$ (nonlinear activation functions or neurons).

3: Obtain the weights $\boldsymbol{\vartheta}\left(k_{\mathrm{c}}\right)$ by the solution of the following optimization problem

$$
\boldsymbol{\vartheta}\left(k_{\mathrm{c}}\right)=\arg \min _{\boldsymbol{\vartheta}\left(k_{\mathrm{c}}\right)} \frac{1}{2} \sum_{\ell=1}^{k_{\mathrm{c}}}\left(J_{\ell}-\boldsymbol{\vartheta}\left(k_{\mathrm{c}}\right)^{\top} \boldsymbol{\phi}_{\ell}^{\left(k_{\mathrm{c}}\right)}\right)^{2}
$$

4: Generate $\Lambda$ random perturbations

$$
\Delta \boldsymbol{\theta}^{(p)}\left(k_{\mathrm{c}}\right)=\alpha\left(k_{\mathrm{c}}\right) \boldsymbol{\delta}^{(p)}\left(k_{\mathrm{c}}\right), \quad p \in\{1,2, \ldots, \Lambda\}
$$

where $\alpha\left(k_{\mathrm{c}}\right)=\alpha_{0} /\left(\alpha_{0}+k_{\mathrm{c}}\right)$ is a time-decaying stepsize and $\boldsymbol{\delta}^{(p)}\left(k_{\mathrm{c}}\right)$ are zero-mean Gaussian random vectors.

5: Produce $2 \Lambda$ new candidate vectors of parameters

$$
\boldsymbol{\theta}^{( \pm p)}\left(k_{\mathrm{c}}+1\right)=\boldsymbol{\theta}^{*}\left(k_{\mathrm{c}}\right) \pm \Delta \boldsymbol{\theta}^{(p)}\left(k_{\mathrm{c}}\right)
$$

where $\boldsymbol{\theta}^{*}\left(k_{\mathrm{c}}\right)$ is the "best" set of tunable parameters until the $k_{\mathrm{c}}$-th experiment, i.e. the one with the best performance so far.

6: Project all the candidate vectors $\boldsymbol{\theta}^{( \pm p)}\left(k_{\mathrm{c}}+1\right)$ to the permissible domain $\Theta=\left[\boldsymbol{\theta}_{\min }, \boldsymbol{\theta}_{\max }\right]$. 
7: Evaluate the effect of each candidate vector to the system performance by using the approximator $\hat{J}^{\left(k_{\mathrm{c}}\right)}$, i.e.

$$
\hat{J}_{( \pm p)}^{\left(k_{\mathrm{c}}\right)}=\boldsymbol{\vartheta}\left(k_{\mathrm{c}}\right)^{\top} \boldsymbol{\phi}^{\left(k_{\mathrm{c}}\right)}\left(\boldsymbol{\theta}^{( \pm p)}\left(k_{\mathrm{c}}+1\right), \overline{\mathbf{x}}\left(k_{\mathrm{c}}+1\right)\right)
$$

where $\overline{\mathbf{x}}\left(k_{\mathrm{c}}+1\right)$ is an estimate of the external disturbances $\mathbf{x}$ for the next experiment $k_{\mathrm{c}}+1$.

8: Pick the vector that corresponds to the best estimate, i.e.

$$
\boldsymbol{\theta}\left(k_{\mathrm{c}}+1\right)=\arg \min _{\boldsymbol{\theta}^{( \pm p)}\left(k_{\mathrm{c}}+1\right)} \hat{J}^{\left(k_{\mathrm{c}}\right)}\left(\boldsymbol{\theta}^{( \pm p)}\left(k_{\mathrm{c}}+1\right), \overline{\mathbf{x}}\left(k_{\mathrm{c}}+1\right)\right)
$$

to determine the set of parameters $\boldsymbol{\theta}\left(k_{\mathrm{c}}+1\right)$ to be applied at the next period $k_{\mathrm{c}}+1$.

The number of lanes for through traffic varies from 2 to 5 and the free flow speed is 45 kilometers per hour. Traffic lights at signalized intersections are operating on multi-phase fixed-time plans with constant (but not equal) cycle lengths $\sqrt[5]{5}$ For the simulation experiments, typical loop-detectors have been installed around the middle of each network link. The OD-based demand that is used for the simulations consists of 123 origin centroids and 132 destination centroids and provides a good replication of real life conditions as it generates realistic traffic congestion patterns in the network. The duration of the simulation is 5 hours including a 15 minutes warm-up period. In the no control case (where a set of the real fixed-time plans of the city are applied to the intersections) the network faces some serious congestion problems, with queues spilling back to upstream intersections. Note, that drivers adapt to the traffic conditions through a C-Logit route choice model (standard module of the simulator) that is activated every 3 minutes, therefore the distribution of demand into the network is more realistic. Previous works (Gayah and Daganzo, 2011b; Mahmassani et al., 2013; Yildirimoglu et al., 2015) have shown for different models and network configurations that driver adaptivity

\footnotetext{
${ }^{4}$ Source: https://maps.here.com/.

${ }^{5}$ Intersections that are considered for perimeter flow control have different constant cycle durations; these durations are not affected here (i.e. only the durations of green phases are changed) in order to maintain the coordination.
} 


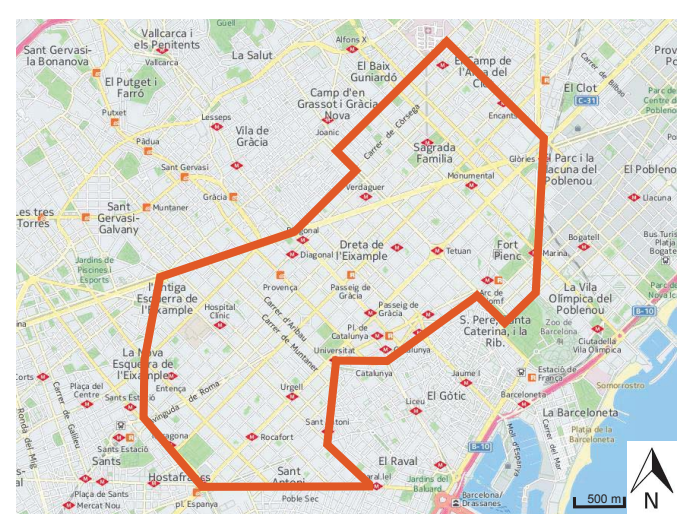

(a)

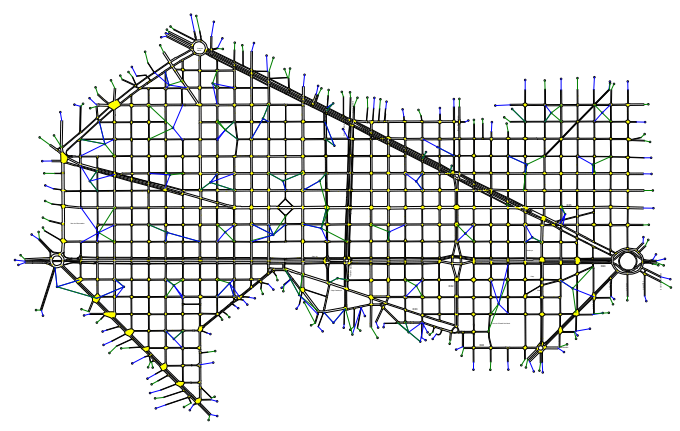

(c)

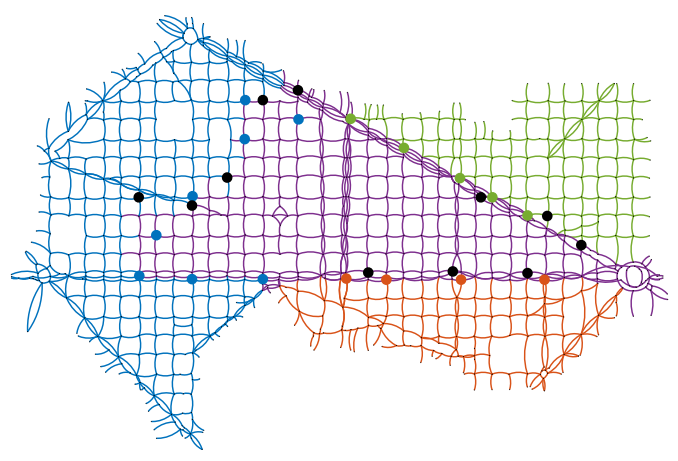

(b)

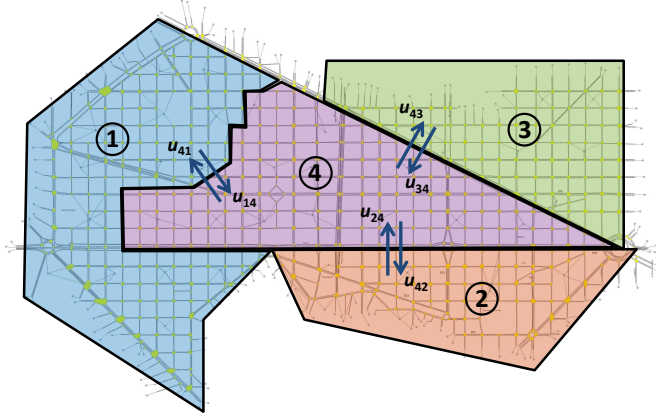

(d)

Figure 3: The test site of Barcelona, Spain: (a) Map of the studied areat (b) results of the clustering algorithm and choice of controlled intersections; blue circles correspond to intersections belonging to $u_{14}$, red to $u_{24}$, green to $u_{34}$ and black to $u_{4 j}, j=1,2,3 ;$ (c) Aimsun model of the network (blue lines connect the centroids to origins and green to destinations); (d) representation of the simulation model partitioned in four regions and the control variables.

increases the performance of large-scale networks and decreases hysteresis loops in the MFDs, which is closer to real-life observations. Note that the developed controller does not utilize any information related to OD; only loop detector data is required.

\subsection{Network partitioning and macroscopic fundamental diagrams}

Traffic congestion in the city of Barcelona is unevenly distributed, creating multiple pockets of congestion in different areas of the network. As MFD depends on the distribution of link densities (occupancies, speeds), partitioning 
heterogeneously loaded cities with uneven distribution of congestion into homogeneous regions is a possible solution to take advantage of well-defined MFDs. In fact, the outflow of the network is a function of both average and variance of link densities. Since traffic conditions are spatially correlated in adjacent roads and congestion propagates from adjacent links, describing the main pockets of congestion in a city with a small number of clusters without the need for detailed information in every link of the network is conceivable. By partitioning, we aim to group spatially-connected links with close density values within a cluster, which increases the network flow for the same average density. Spatial connectivity is a necessary condition that makes feasible the application of perimeter control strategies. The partitioning algorithm used in this study is an optimization framework called "Snake" algorithm (Saeedmanesh and Geroliminis, 2016), which considers heterogeneity index as a main objective function and contiguity is forced explicitly by imposing constraints. This approach requires the desired number of clusters as a predefined input and it obtains the optimal number of clusters by evaluating heterogeneity metric for different number of clusters. By applying this algorithm, the network of Barcelona is partitioned into 4 homogeneous regions that are shown in Figure 3(d), This partitioning simplifies the network dynamics as there is no need for routing information/decisions (i.e. due to the configuration of the regions there is only one choice to move from one region to another). Note, however, that the methodological control approach proposed in the previous sections can be applied to any arbitrary partitioning outcome.

The simulation is first executed with the fixed-time signal plans of the city to obtain the data needed for the control design. Figure 4(a) presents the production MFD for the whole network for the first two hours of the simulation (onset of congestion) and ten different replications, each one with a different random seed that affects the stochastic parameters of the microsimulator. Each point corresponds to the aggregated measurements of all the detectors and the time interval is $90 \mathrm{sec}$ (i.e. equal to the control interval $T$ ). The network MFD has low scatter and reaches the congested regime (production reduces from 4500 to $2000 \mathrm{veh} \cdot \mathrm{km} / T$ ). The production of each region separately for all replications (again for the first two hours) is displayed in Figure 4(b). Note that region 2 is the only region that does not get congested, whereas the rest of the regions get states with increased accumulations and decreased productions. Region 4 is the only one that has common boundaries with all other regions. Figures 4(c) and 
4(d) demonstrate the MFDs for the transfer flows $u_{41}^{\mathrm{NC}} M_{41}, u_{42}^{\mathrm{NC}} M_{42}, u_{43}^{\mathrm{NC}} M_{43}$ and the trip completion rate $M_{44}$ as third degree polynomial functions of the region accumulation $n_{4}$. The variable $u_{\mathrm{ij}}^{\mathrm{NC}}$ denotes the fixed-time control applied to the network of Barcelona between region $i$ and $j$. It is computed by summing the $g_{z} S_{z} / C_{i j} S_{i j}$ factors for all the signalized boundary links $z$ of the $i \rightarrow j$ movement. This derives a constant term for each movement, i.e. $u_{\mathrm{ij}}^{\mathrm{NC}}$, which is then used to scale the transferring MFDs and get the sending flows $M_{i j}$ that are used in the model. The data for the first three transfer flows of the figures is extracted from the same 10 replications by analysing all vehicle trajectories, but can be also obtained (in simulation and real life) from the flow measurements of all the loop detectors along the boundaries between any two regions. Finally, the trip completion rate $M_{44}$ is computed for all the measurement points by equation (2).

The MFDs have relatively low scatter and the fitted functions are utilized by the model in order to derive the LQI multivariable regulator. This modelbased control design phase assumes that these functions do not depend on the control decisions (simulation data before and after control validates this assumption) and are only a function of the accumulation of the sending region (something that is demonstrated by the simulation data in Figures 4(c) and $4(\mathrm{~d})$. The feedback nature of the regulator and the online tuning/optimization by AFT can correct possible discrepancies between modelling assumptions and real world (or microsimulation in this case study).

\subsection{Simulation set-up and offline design of $L Q I$}

The network partitioning presented in the previous section derives $M=6$ control and $N=4$ state variables (i.e. $\mathbf{u}=\left[\begin{array}{llllll}u_{14} & u_{24} & u_{34} & u_{41} & u_{42} & u_{43}\end{array}\right]^{\top}$ and $\left.\mathbf{n}=\left[\begin{array}{llll}n_{1} & n_{2} & n_{3} & n_{4}\end{array}\right]^{\top}\right)$. The duration of the simulation is 5 hours, where the first 2 hours represent the onset of congestion and the rest 3 hours (offset of congestion) are used to make sure that the network is empty of vehicles at the end of the simulation period and the evaluation metrics are comparable. The simulation step is set to $0.5 \mathrm{sec}$ and the multivariable PI regulator is applied every $T=90 \mathrm{sec}$. The control decisions (after modified to satisfy the operational constraints) are forwarded for application to 28 signalized intersections (out of 600 in the network) which are all across the boundaries of region 4. As shown in Figure 3(b), there are 8 intersections for applying $u_{14}$ (blue circles), 4 for $u_{24}$ (red circles), 5 for $u_{34}$ (green circles), 5 for $u_{41}, 3$ for $u_{42}$ and 3 for $u_{43}$. All the intersections that control the outflow of region 4 are indicated in Figure 3(b) with black circles although they 


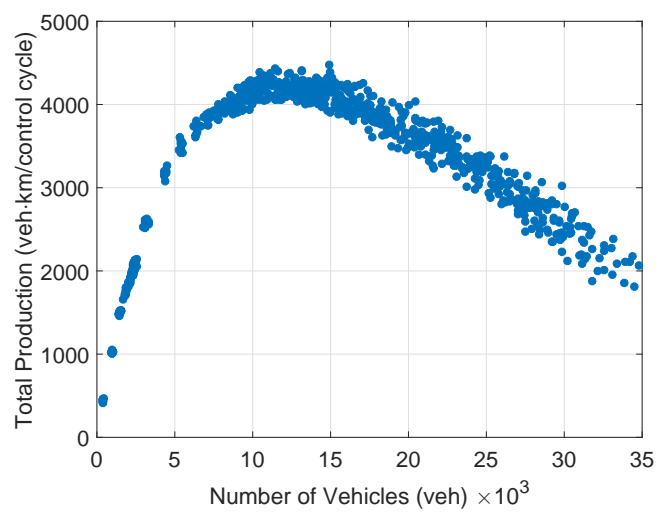

(a)

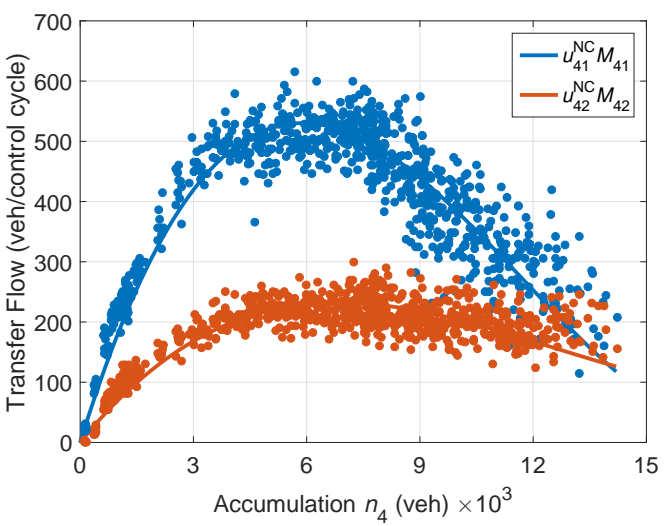

(c)

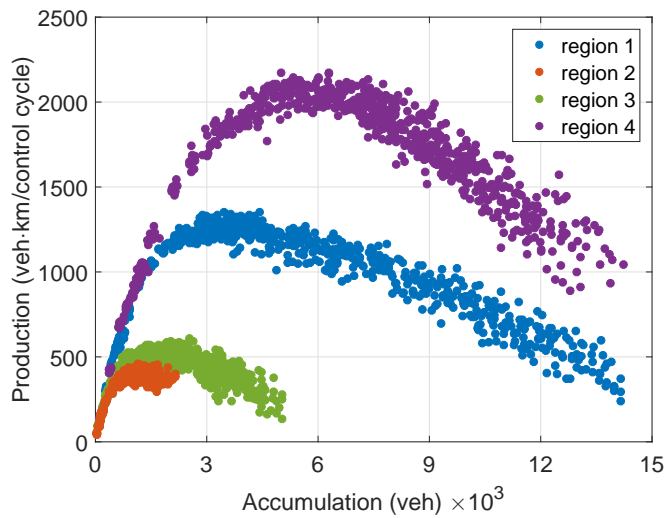

(b)

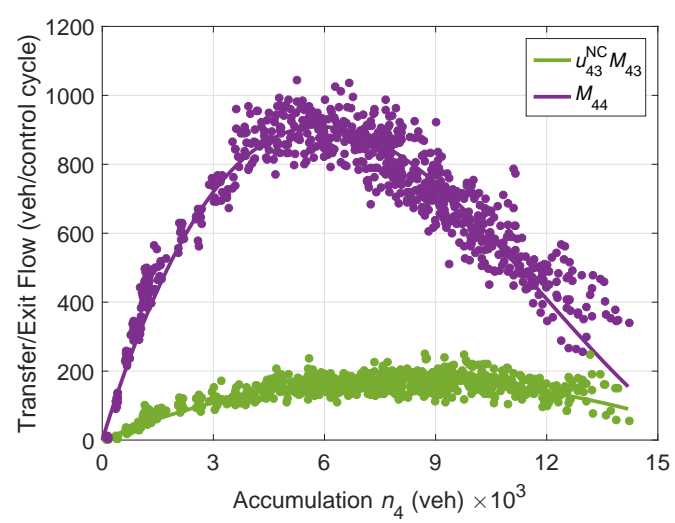

(d)

Figure 4: (a) Production MFD for the whole network; (b) production MFD for each region of the network; (c) transfer flows $u_{41}^{\mathrm{NC}} M_{41}\left(n_{4}\right), u_{42}^{\mathrm{NC}} M_{42}\left(n_{4}\right)$ MFDs for region 4; (d) transfer flow $u_{43}^{\mathrm{NC}} M_{43}\left(n_{4}\right)$ and internal trip completion $M_{44}\left(n_{4}\right)$ MFDs for region 4.

correspond to different control variables. Their location at the borders of region 4 indicates the control variable in which they belong. The signal plans of the aforementioned intersections, and more precisely their operational constraints (e.g. minimum time allocated for pedestrian phases), determine the corresponding minimum and maximum permissible rates for the control variables $\mathbf{u}$ (constraint (5)). The derived $\mathbf{u}_{\min }, \mathbf{u}_{\max }$ applied at the simulation are given by $\mathbf{u}_{\min }=\left[\begin{array}{llllll}0.069 & 0.077 & 0.061 & 0.068 & 0.077 & 0.077\end{array}\right]^{\top}$ and $\mathbf{u}_{\max }=$ $\left[\begin{array}{llllll}0.827 & 0.695 & 0.604 & 0.824 & 0.81 & 0.76\end{array}\right]^{\top}$, which are computed by using the minimum and maximum green phases duration of the 28 intersections. 
For the LQI methodology different linearization set-points $\hat{\mathbf{n}}$ are investigated, which lead to different gain matrices that influence the performance of the regulator. The selection of appropriate set-points $\hat{\mathbf{n}}$ for a multi-region system is not straightforward, as at a given time the states of neighbouring regions may be at different regimes of the MFD (i.e. congested or uncongested); hence the linear approximations of the model may not be reliable. Here, many different vectors are used for $\hat{\mathbf{n}}$ (spanning various region states ${ }^{6}$ ) and the results of the simulations provide observations that can be used as initial samples for the learning procedure of AFT algorithm. The matrix $\mathbf{C}$ that provides the state errors and contributes to the integral part of the regulator is set equal to $\mathbf{C}=\left[\begin{array}{llll}1 & 0 & 0 & 0 \\ 0 & 0 & 0 & 1\end{array}\right]$, which means that only errors measured for regions 1 and 4 are considered. Since these two regions are more important (masters) the objective criterion minimizes the integral of their state error $n_{i}(k)-\hat{n}_{i}, i=1,4$. The regions 2 and 3 are not included in the integral part, which means that this design does not guarantee zero steady-state error for these regions (slaves). Nevertheless, this configuration renders the dynamical system fully controllable. Finally, the diagonal weighting matrices $\mathbf{Q} \in \mathbb{R}^{4 \times 4}$, $\mathbf{R} \in \mathbb{R}^{6 \times 6}$ and $\mathbf{S} \in \mathbb{R}^{2 \times 2}$ are chosen after trial-and-error experiments by studying the behaviour of the controller. Physically speaking, these weights depend upon the order of magnitude of each variable and also the weight of each term on the objective criterion. To this end, many different sets were tested until achieving a satisfactory control behaviour. Specifically, the diagonal elements of $\mathbf{Q}$ are chosen according to the maximum accumulation of each region (albeit with different weights), i.e. $Q_{i i}=1 / n_{i, \max }$ for $i=1,4$ and $Q_{i i}=5 / n_{i, \max }$ for $i=2,3$; the diagonal elements of $R, S$ are chosen $R_{i i}=500, S_{i i}=10^{-6}$ for $i=1,2,3,4$.

\subsection{Control scenarios}

Different control scenarios are investigated. The base case is the no control (NC) scenario where one set of the real well-tuned fixed-time plans of the city is applied to the network. The performance of different control approaches is evaluated according to the improvement that they can accomplish compared

\footnotetext{
${ }^{6}$ Different levels of congestion are considered for each region (e.g. $0.8 n_{\mathrm{cr}}, 0.9 n_{\mathrm{cr}}, 1.1 n_{\mathrm{cr}}$, $1.2 n_{\mathrm{cr}}$, where $n_{\mathrm{cr}}$ is the critical accumulation of the region) and many combinations of the states are used to produce the set-points $\hat{\mathbf{n}}$ and derive linearizations of the corresponding MFDs.
} 
to the base case. One option is to apply AFT alone starting from a fixedtime controller and trying to converge to parameters that will improve the performance (pure data-driven approach). Another alternative is to use the model-based PI controller that is derived from LQI optimization methodology for different linearization points. Finally, the two aforementioned approaches can be combined into one by using LQI for the first iterations (days) and then switch to the online adaptation of the parameters by exploiting the knowledge of the first iterations (LQI/AFT). As demonstrated later a combination of LQI for the first iterations with online adaptation provides the best performance for the network.

Another scenario that is tested is to protect only one region of the network. The simulation results reveal that a single region perimeter control, even if it is carefully designed, is unable to bring the system to a desired state and congestion is high. This significantly highlights the importance of careful integration of concepts consistent with the physics of traffic in the methodological and control framework. Region 4 has been selected to be protected (as it attracts the highest demand from the origins compared to the other regions) and the total inflow and outflow to this region is regulated in the perimeter by a simple I-type regulator which has the following form

$$
\mathbf{u}_{4}(k)=\mathbf{u}_{4}(k-1)-\mathbf{K}_{4}\left[n_{4}(k)-\hat{n}_{4}\right]
$$

where $\mathbf{u}_{4} \in \mathbb{R}^{2 \times 1}$ contains the two decision variables that regulate the inflow and outflow respectively and there is only one state variable $n_{4}$, i.e. the accumulation of region 4 . Similarly to $(15)$, the vector $\mathbf{K}_{4}^{2 \times 1}$ comprises the gains of the regulator (with $K_{4}(1) \in \mathbb{R}^{+}$and $K_{4}(2) \in \mathbb{R}^{-}$the gains for inflow and outflow, respectively). This single region (SR) control scenario utilizes the same 28 intersections displayed in Figure 3(b) at the boundary of region 4 and its objective is to operate the accumulation around the desired point in order to prevent congested states for this region. In principle, this feedback regulator increases/decreases the inflow/outflow of the region based on the difference between the current accumulation and the desired one (when inflow is decreased because of high accumulation $n_{4}(k)$, outflow increases as $K_{4}(1)$ and $K_{4}(2)$ have different signs). It is activated/deactivated whenever the state $n_{4}(k)$ is higher/lower than $\left.0.8 \hat{n}_{4}\right]^{7}$. As demonstrated later, such a

\footnotetext{
${ }^{7}$ This value has been found after a manual trial-and-error procedure; AFT has been also used to optimize this parameter but the performance could not be improved any further.
} 
controller is not successful in decreasing congestion and bringing region 4 and the overall network in better states. The main reasoning about this is related to spillbacks to the other regions combined with high uncontrolled inflow in region 4. The results of all different control approaches are discussed in the next section.

\subsection{Simulation results}

This section presents the results obtained by the simulation experiments. First, AFT is applied online without any prior knowledge of the system and tries to optimize the regulator parameters. This is a pure data-driven approach and no model is utilized for the controller. AFT runs for 100 iterations starting from an initial point where $\mathbf{K}_{\mathrm{P}}=\mathbf{K}_{\mathrm{I}}=\mathbf{0}_{6 \times 4}$. For these values the regulator (15) operates as a fixed-time policy and this point is equivalent to the $\mathrm{NC}$ case (i.e. the actual fixed-time plans of the city are applied). The initial values for the set points $\hat{\mathbf{n}}$ are obtained from the production MFDs of the NC case (Figure 4(b) and are equal to $\hat{\mathbf{n}}=[3600,1400,2000,6000]^{\top}$. The performance index of AFT (i.e. the objective function $J$ that tries to minimize) is selected to be the total delay of the system. In each iteration the whole simulation of 5 hours runs with the same parameters and the multivariable regulator is activated/deactivated according to the predefined thresholds $n_{i \text {,start }}$ and $n_{i, \text { stop }}{ }^{8}$ At the end of the simulation AFT is called to calculate the new values of $\mathbf{K}_{\mathrm{P}}, \mathbf{K}_{\mathrm{I}}, \hat{\mathbf{n}}$ to be used in the next iteration.

Figure 5(a) presents the evolution of the average system delay (measured in sec $/ \mathrm{km}$ ) over the iterations of the algorithm. For the first iterations the system performance is extremely ineffective, leading to values of delay three times higher than the NC case. This "spiky" behaviour of the algorithm (also reported in Kouvelas et al. (2015)) occurs due to the fact that in the first iterations there are not many samples (i.e. no prior knowledge of system performance for different controllers) and the approximator cannot learn from previous experiments. As the number of iterations increases the learning process becomes better and the objective function exhibits a convergent behaviour, leading to results that improve the initial fixed-time controller (red dashed line in Figure 5(a)). Nevertheless, such an approach might be difficult to be tested in a real framework as the first few days the control will be catastrophic for the system.

\footnotetext{
${ }^{8}$ The thresholds are selected according to the critical accumulations of each region and $n_{i, \text { stop }}=n_{i, \text { start }}=0.8 n_{i, \text { cr }}$.
} 
To overcome this "spiky" behaviour, AFT is combined with the regulators that are derived by application of LQI methodology to the linearized model. More specifically, different LQI regulators are generated (for different linearization set-points) and are simulated to the network for the first iterations. Then, AFT is applied for online optimization, exploiting the knowledge of all the conducted simulations (samples of performance $J$ for different values of $\left.\mathbf{K}_{\mathrm{P}}, \mathbf{K}_{\mathrm{I}}, \hat{\mathbf{n}}\right)$. The results are presented in Figure $5(\mathrm{~b})$. In the first 13 iterations different multivariable PI regulators are applied that are all obtained by the solution of LQI. The figure displays the average value of the performance index (average delay) for these 13 iterations with a magenta dashed line. The blue solid line presents the evolution of delay over AFT iterations, while the red dashed line indicates the delay of $\mathrm{NC}$ case. The algorithm applies different perturbations of the parameters until it converges. Note by comparing Figures 5(a) and 5(b) (vertical axes have different range of values for visibility purposes) that the high values of delays in the first iterations are avoided in 5(b), which allows for a potential real life implementation of the approach (compared to the first problematic iterations of the classical AFT). Note also, that applying AFT with some good initial iterations (coming from LQI methodology) facilitates the convergence of the system to better and smoother solutions, $378 \mathrm{sec} / \mathrm{km}$ vs. $331 \mathrm{sec} / \mathrm{km}$ (compare Figures 5(a) and 5(b) after iteration 70). In previous applications of AFT algorithm (see e.g. Kouvelas et al. (2011a)) a fairly good initial point was provided for the tuneable parameters (i.e. predefined values based on particular problems experience) and as a consequence there was no need to generate some "good" initial iterations. Another technique that has been used is to apply relatively small stepsizes $\alpha_{0}$ in Step 4 (see Algorithm 1) in order to prevent the "spiky" behaviour of the first iterations (especially in field implementations), but this has the shortcoming that the algorithm may stack in local minima and not improve the performance.

Table 1 presents some quantitative results about the simulations. First, the performance index of AFT (average delay ${ }^{9}$ ) for all different approaches is reported. Taking NC as the base case, the delay decreases by about $3.4 \%$ when the SR control is applied. In this scenario, region 4 is protected by the controller, but due to the excessive demand and the propagation of

\footnotetext{
${ }^{9}$ Since the network is empty at the end of the simulation period (i.e. all simulations serve the same number of vehicles), average and total delay represent the same metric.
} 


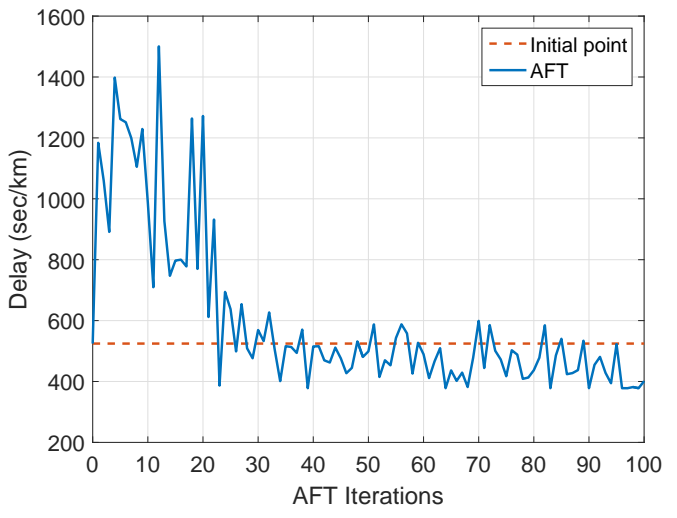

(a)

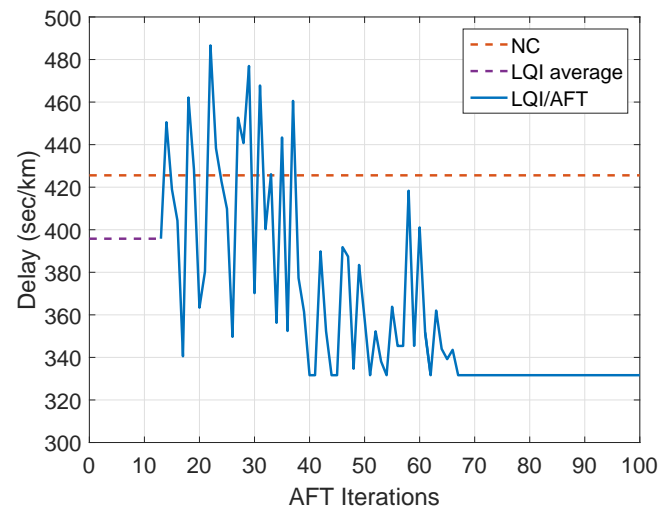

(b)

Figure 5: (a) Evolution of network delay for the 100 iterations of AFT; (b) AFT convergence after running the algorithm with 13 initial points from LQI.

Table 1: Performance index of different approaches; (b) evaluation criteria for $\mathrm{NC}$ and $\mathrm{BC}$ simulations.

\begin{tabular}{lllllll}
\hline & NC & SR & LQI & AFT & LQI/AFT & Units \\
\hline Delay & 425.57 & 411.19 & 395.79 & 378.01 & 331.64 & $\mathrm{sec} / \mathrm{km}$ \\
Criterion (14) & 1.1984 & 0.6348 & 0.8144 & 0.6822 & 0.4556 & $\mathrm{veh} \times 10^{3}$ \\
\hline \multirow{2}{*}{ Improvement } & - & 3.38 & 7 & 11.18 & 22.07 & \\
& - & 47.04 & 32.05 & 43.08 & 61.99 & $\%$ \\
\hline
\end{tabular}

congestion to other regions the relative improvement is insignificant. Clearly, protecting the region with the higher attraction of destinations is not enough for the overall network performance. When the multivariable LQI regulator is applied to control all the regions the improvement rises to $7 \%$. It should be emphasized that this is the average delay of the 13 points obtained for different linearization set-points and this approach deserves further investigation (i.e. how one could choose proper $\hat{n}_{i}$ without a fine-tuning method similar to AFT). In the case that AFT is applied without any prior knowledge the improvement is about $11.2 \%$ (for the best run); furthermore this approach has the "spiky" behaviour during the first iterations, and also, even after convergence the performance for different runs is not stable (see Figure 5(a)]. By using the LQI points for the first iterations and then applying AFT we obtain a stable convergent behaviour and an improvement of some $22.1 \%$. Then, the value of 
criterion (14) is reported for all control approaches. It should be noted that only the first and third terms of the quadratic criterion are computed, as the second term (i.e. variations form nominal control values) cannot be defined for some of the scenarios (e.g. NC case). The value of the objective function is calculated for a time window that most of the controllers are active, i.e. from time $t=1800 \mathrm{sec}$ until $t=10800 \mathrm{sed}^{10}$. According to this criterion SR outperforms LQI and AFT approaches while BC is again the most effective.

As a mater of fact the single region (SR) control does not manage to alleviate congestion, even if it has been designed properly. This highlights that a method that controls only the external boundary of a network and estimates the delays outside this boundary with a virtual queue approach, might disregard significant traffic phenomena related to delays and spillbacks, especially if some of these movements carry substantial portions of the traffic demand. Figure 6 provides some more insights about the performance of SR controller. The goal of the feedback regulator 22 is quite straightforward (i.e. it tries to keep region 4 around the desired point and does not take into consideration the states of the other regions) and its behaviour (e.g. aggressive or conservative actions, oscillations) depends on the gains $\mathbf{K}_{4}$. Several experiments were carried out for the SR regulator and according to the simulations results the gains $\mathbf{K}_{4}=\left[\begin{array}{ll}0.008 & -0.008\end{array}\right]^{\top}$ were found to have a satisfactory performance (e.g. non oscillatory behaviour, empty network at the end of the simulation). It should be noted that there are many values of these gains for which the network faces severe spillbacks (mostly between regions 1 and 4) that take a lot of time to be resolved. For the simulation of the aforementioned gains (which has a total delay improvement of 3.38\%), Figure 6(a) presents the evolution of accumulation in region 4 . Note the small improvement over the $\mathrm{NC}$ case even if the regulator does not achieve the target point due to excessive internal demand, operational constraints on the control variables and spillbacks that affect the ordered flows. Nevertheless, the total delay for region 4 (area between the two trajectories) is reduced significantly. Figure 6(b) displays the control actions of the regulator which

\footnotetext{
${ }^{10}$ Note that the compared controllers are not all deactivated at the same time, as this is done based on measurements from the simulation and the evolution of congestion is not the same. Here, a window of 100 control cycles has been chosen because most of the controllers are active during all this period. Finally, the $\hat{\mathbf{n}}$ vector of Section 5.5 is used in the calculations for all the scenarios (for the sake of comparison), although the controllers are designed for different set-points (or tuned with AFT).
} 


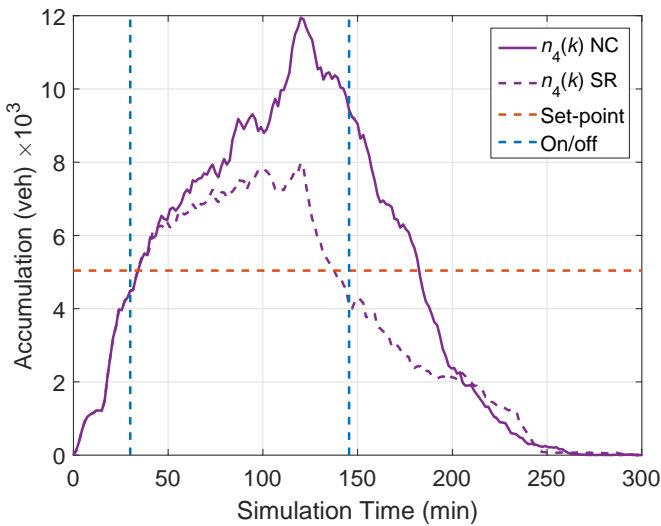

(a)

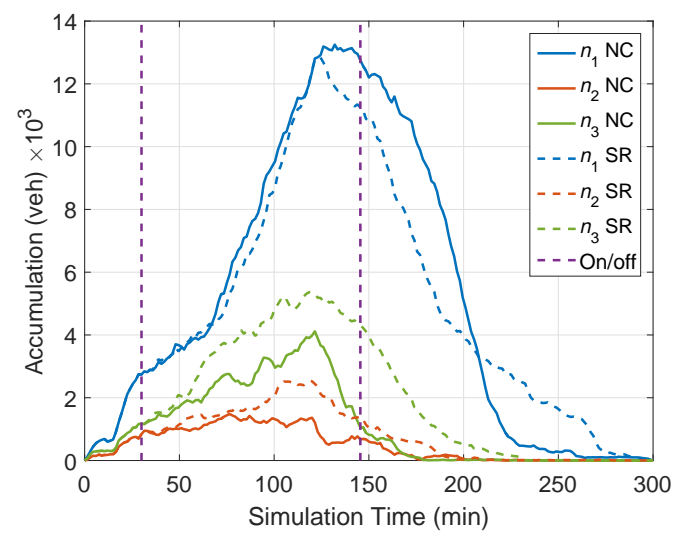

(c)

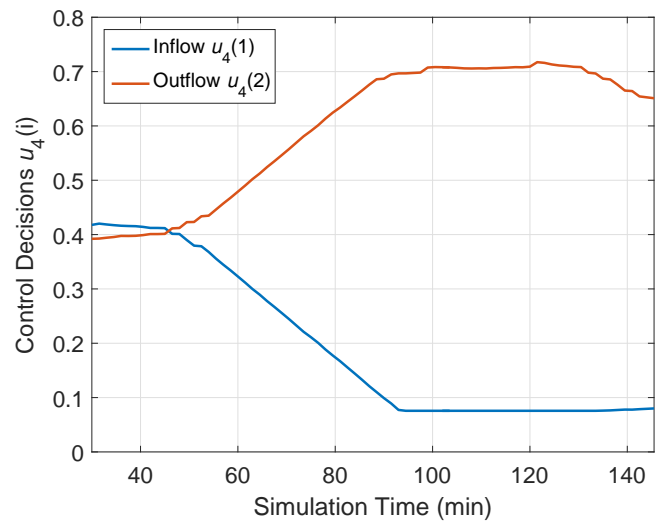

(b)

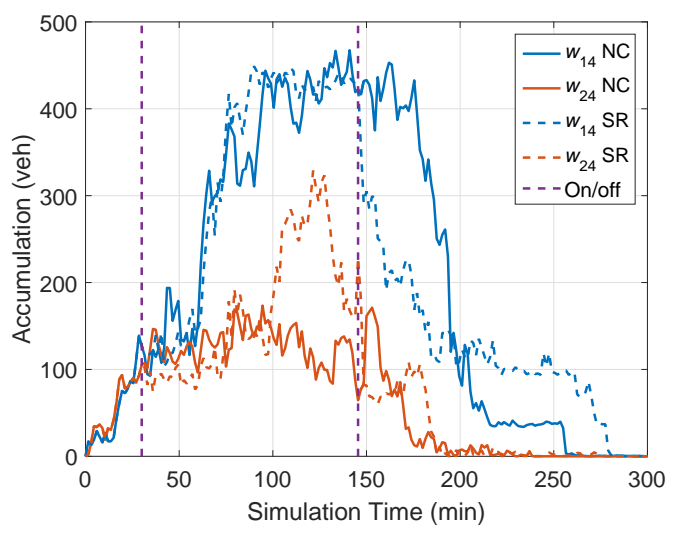

(d)

Figure 6: (a) Evolution of accumulation of region 4 for NC and SR cases and set-point of single region controller; (b) control decisions in the boundaries of region 4 for SR case (regulated inflow and outflow); (c) evolution of accumulations for the other 3 regions of the network for $\mathrm{NC}$ and SR cases; (d) total vehicles waiting to enter region 4 from region 1 and $2\left(w_{14}\right.$ and $w_{24}$ respectively) over time for NC and SR cases.

is activated at minute 30 and deactivated at minute 145.5. As expected, the inflow decreases gradually to the minimum value (and the outflow increases to maximum) because accumulation in region 4 is higher than the target for a long period. This is beneficial for region 4 but also does not cause many problems to the other regions (see accumulations in Figure 6(c)). Region 1 is also improved compared to $\mathrm{NC}$ for most of the simulation time, whereas regions 2 and 3 experience some longer delays. 
By comparing the accumulations of regions 1 and 4 for SR and NC after time 200 min (Figures 6(a) and 6(c)) we observe that the SR case experiences higher delays. This is a result of local spillbacks in an area that spans these two regions and can be also noticed in Figures 6(d). This figure presents the total number of vehicles inside the links that are controlled in the boundary between regions 1 and $4\left(w_{14}\right)$ and 2 and $4\left(w_{24}\right)$ over time, i.e. vehicles that are waiting to enter region 4 from regions 1 and 2 respectively. It is the stripe of links around the boundary (31 roads). In the boundary between 1 and 4 the SR controller has about the same inflow with $\mathrm{NC}$ until the time that the controller is deactivated. This is achieved with much smaller green duration because in the $\mathrm{NC}$ case there are spillbacks in region 4 that prevent vehicles from entering. Once the controller is deactivated (i.e. both cases have the same fixed-time plan but the accumulation in region 4 is much lower in SR) many more vehicles can enter region 4 and $w_{14}$ decreases dramatically. However, this has a negative effect later in time as there is a blocking that delays vehicles in $w_{14}$ (which also exists in the $\mathrm{NC}$ case but for fewer vehicles and shorter time period; see Figures 6(d) after time $200 \mathrm{~min}$ ). In the boundary between regions 2 and 4 things are more clear, with $w_{24}$ being higher for SR when the controller is active (due to reduced green duration) and no blocking occurrence after the deactivation of the controller actions. The number of vehicles in the boundary between 3 and 4 (not presented here) has a similar pattern to $w_{24}$. In conclusion, it should be emphasized that this is the best SR controller (of this type) that we obtained after a tedious trial-and-error procedure. It is clear that by only protecting region 4 there are not many actions that someone could do to improve the total network delay.

The qualitative characteristics of the best controller (BC) that is obtained after AFT convergence are further investigated in an attempt to interpret its behaviour. Figure 7(a) illustrates the time series of accumulations for all regions and for NC (solid lines), BC (dashed lines), respectively. The controller achieves to maintain the system in better states, i.e. the accumulations of regions 1 and 4 are substantially improved while regions 2 and 3 are slightly deteriorated, as they try to support regions 1 and 4 that have a higher attraction of trips. The integral of the areas between the solid and dashed lines corresponds to the improvement/deterioration of the total delay. The space-mean speed of each region (i.e. $\nu_{i}(k)=P_{i}(k) / n_{i}(k)$ ) over the simulation time is illustrated in Figure $7(\mathrm{~b})$ from $t=20 \mathrm{~min}$ to $t=220 \mathrm{~min}$. Note that speed increases for regions 1 and 4 shortly after the controller is activated and less number of vehicles observed in these regions, while regions 2 and 3 


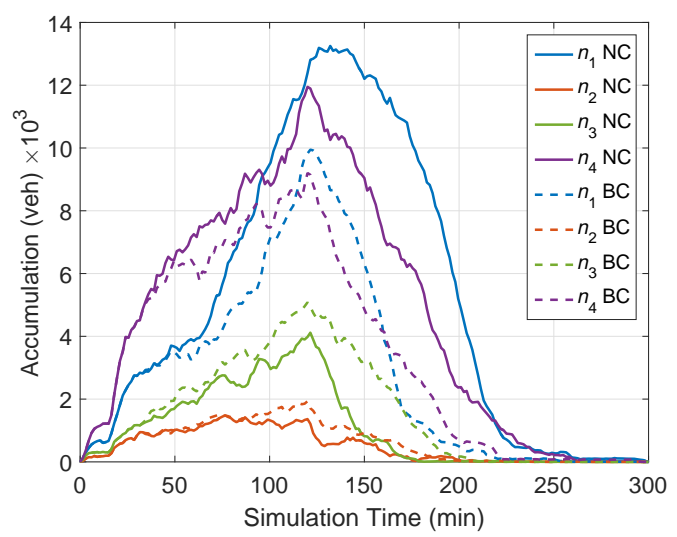

(a)

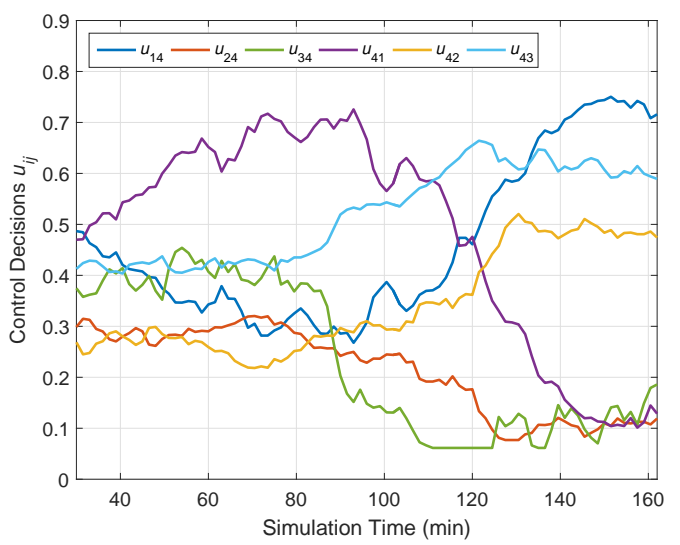

(c)

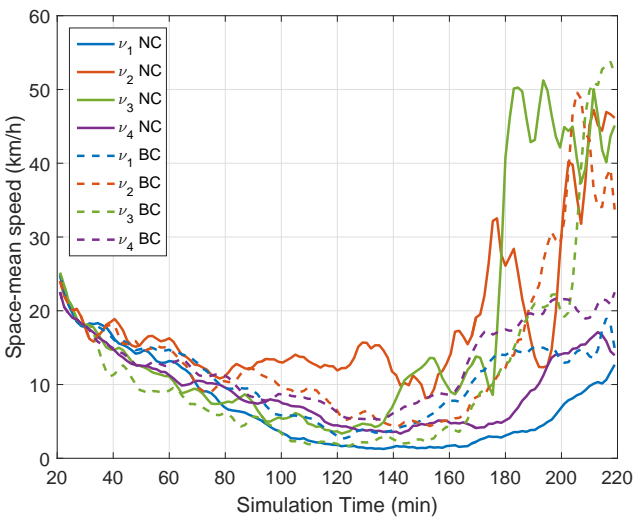

(b)

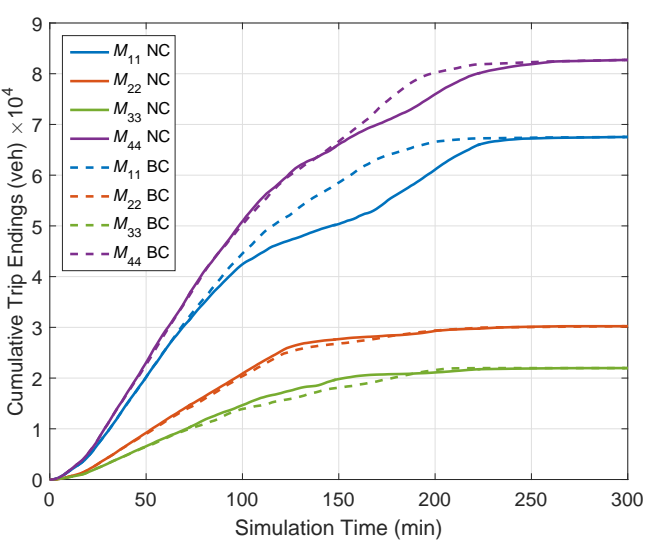

(d)

Figure 7: (a) Time series of regions accumulations for $\mathrm{NC}$ and $\mathrm{BC}$; (b) time series of regions space-mean speeds for $\mathrm{NC}$ and $\mathrm{BC} ;(\mathrm{c})$ control decisions over simulation time for the $\mathrm{BC}$ case; (d) cumulative trip endings over simulation time for $\mathrm{NC}$ and $\mathrm{BC}$.

are holding more vehicles due to perimeter control restrictions. Figure $7(\mathrm{c})$ displays the trajectories of the control variables for the BC case. The controller is activated after 30 minutes and stays active for 2 hours and 12 minutes. It is clear from the figure that the antagonistic control variables $u_{14}$ and $u_{41}$ or $u_{34}$ and $u_{43}$ exhibit some kind of inverse variation (i.e. when the one increases the other decreases and vice versa) and this is more clear when region 4 gets congested (after minute 80). This also happens for variables $u_{24}$ and $u_{42}$ but to a smaller extend. Figure $7(\mathrm{~d})$ presents the cumulative number of trip 
endings (i.e. vehicles reaching their destination) during the simulation for every region. It is clear that $\mathrm{BC}$ improves the throughput of regions 1 and 4 (masters) while regions 2 and 3 have less throughput (slaves). In total, BC manages to serve more vehicles than $\mathrm{NC}$ for the same simulation time, and, as a result, the dissipation of network congestion happens much earlier in the BC case. Note that while speed is higher and accumulation is lower after $t=30 \mathrm{~min}$ for region 4 , trip endings increase compared to $\mathrm{NC}$ case only after $t=150 \mathrm{~min}$. The main reason is that during this period there is a large number of trips with external destinations to region 4 that passes through this region (especially with destination in region 1). The transfer flows are significantly higher (see also pattern for $u_{41}$ ) and more trip endings occur earlier in region 1.

Finally, in order to account for the stochasticity of the simulator, 10 replications were carried out (with different random seeds). These replications provide a statistical analysis of $\mathrm{NC}$ and $\mathrm{BC}$ cases for different variations of the stochastic parameters of the software. They can be considered - in an analogy to a real case - as different variations among congested days with similar but not identical demand profiles. Table 2 presents some average quantitative results of the simulation experiments, where $\mathrm{NC}$ and $\mathrm{BC}$ are compared for different metrics obtained by the simulator (the average performance of the 10 replications is presented here). BC outperforms $\mathrm{NC}$ in all evaluation criteria as the applied controller manages to distribute congestion in a better way. More precisely, the improvement of the delay is about $20 \%$ on average, the space-mean speed of the network is increased by about $22.5 \%$ and the time

Table 2: Evaluation criteria for no control (NC) and best controller (BC) simulations (average of 10 replications).

\begin{tabular}{lllll}
\hline Evaluation criteria & $\mathrm{NC}$ & $\mathrm{BC}$ & $(\%)$ & $\mathrm{Units}$ \\
\hline Delay & 514.17 & 410.93 & -20.08 & $\mathrm{sec} / \mathrm{km}$ \\
Space-mean Speed & 6.26 & 7.67 & 22.62 & $\mathrm{~km} / \mathrm{h}$ \\
Stop Time & 426.82 & 333.41 & -21.89 & $\mathrm{sec} / \mathrm{km}$ \\
Mean Queue & 8519.4 & 6358.39 & -25.37 & $\mathrm{veh}$ \\
Mean Virtual Queue & 8619.75 & 7856.77 & -8.85 & $\mathrm{veh}$ \\
Total Travel Time & 70150.94 & 54371.89 & -22.49 & $\mathrm{~h}$ \\
Total Travelled Distance & 419779.67 & 401040.89 & -4.46 & $\mathrm{~km}$ \\
Vehicles Served & 201810 & 201810 & 0 & $\mathrm{veh}$ \\
\hline
\end{tabular}


that a vehicle is stopped (because of congestion or traffic lights) is also $22 \%$ less. The statistics of the queues are also reported in order to demonstrate the improvement of the applied control regarding the virtual queues. Interestingly, the average value of all the network virtual queues over time is reduced by almost $9 \%$ (i.e., drops from 8619.75 to 7856.77 vehicles). The control actions (which are all internal to the network) alleviate traffic congestion - this can be also seen by the $25 \%$ improvement of the mean queue, which corresponds to all the stopped vehicles inside the network over time - and as a consequence they implicitly also reduce the boundary virtual queues (in contrary to previous approaches that significantly increase the boundary queues). In addition, the total travel time is decreased by $22.5 \%$ on average. Note that in both cases all the vehicles are served (as the network is empty at the end of the simulation), though the total travelled distance (production) of NC case is slightly higher (4.46\%). This happens because of the applied driver adaptivity module which makes the vehicles choose longer routes (but with shorter travel times) since the network is heavily congested.

\section{Conclusions and discussion}

A new macroscopic MFD-based model that describes the aggregated dynamics of multi-region systems is introduced. Linear approximations of the model are used to derive optimal multivariable PI feedback regulators (LQI) for perimeter flow control. Furthermore, the performance of the regulator is enriched in real-time by an online adaptive optimization algorithm (AFT). The efficiency of the integrated adaptive control scheme was tested in microsimulation. The studied problem is quite difficult from a control viewpoint, since the boundaries of the network are not controlled and all the inflows coming from the origin links are considered disturbance for the system. As a consequence, it is difficult to regulate the system around the set-point by only controlling the inter-transferring flows between the regions (because of the high disturbances). Nevertheless, it is shown that by considering the spatial and temporal heterogeneity between the regions and integrating a partitioning approach, one can improve the distribution of congestion in the network through perimeter control actions. The simulation results indicate that the integrated control scheme (LQI/AFT) can substantially improve the network performance compared to fixed-time signal plans and previous adaptive type controllers.

As illustrated here, the online application of AFT algorithm without any 
prior observations of the system performance for different control parameters is cumbersome, because of its "spiky" behaviour during the first iterations (e.g. days) of application. To overcome this difficulty, measurements of the system performance can be collected for different controllers that are designed offline by applying the LQI methodology. Then, AFT algorithm is applied to fine-tune the parameters of the multivariable controller (gain matrices and set-points) so as to achieve a desirable performance. The proposed methodology is applicable in real life as it is computationally efficient and it only requires loop detector real-time measurements. Future research directions will deal with investigations about the activation/deactivation time of the controllers for multi-region systems, as well as the possibility of integrating time-dependent parameters (i.e., different gains and set-points in the onset and offset of congestion). These are mutually related problems that can be possibly solved together in real-time.

It should be noted, that strong fluctuations in the demand that create fast evolving transient states, spatial heterogeneity of congestion or route choice effects can influence the trip length distribution of vehicles in the network (and the space-mean trip length); as a result, the ratio of production over outflow (utilized by equation (2) ) might exhibit some variations (Mahmassani et al., 2013; Yildirimoglu and Geroliminis, 2014). If conditions change rapidly however, the outflow-MFD might not provide a good approximation as it is "memoryless", i.e. it ignores the history of the system. If, for instance, there is a discontinuity in the inflow in the uncongested regime, the accumulation increases instantaneously and the outflow predicted by the outflow-MFD increases as well. Intuition suggests that the outflow should only increase after a delay corresponding to the shortest trip duration. While such a model is described in Lamotte and Geroliminis (2016), its complexity makes the integration in a control framework infeasible. As also pointed by Arnott (2013), such a model has an endogenous delay term, which makes its analytical solution very challenging. Also, one of the reviewers of the paper mentioned that in the extreme case that demand patterns follow a Dirac function (with a zero value everywhere except of a single point with value infinity) then the model of equation (2) will produce infinite speeds for some vehicles. Nevertheless, this assumption pushes the model in extreme situations that probably will never occur in real transport networks. An important aspect is that further research is needed to better identify the demand conditions under which the outflow-MFD model might experience significant errors that deteriorate the control efficiency. Nevertheless, we have to point out that 
the perimeter controllers developed here rely on feedback, and thus some discrepancies in the model are not considered to be crucial when feedback frequency (i.e. prediction horizon) is not long.

Another challenging topic is the theoretical analysis of the multi-region nonlinear system with time varying delays, in order to develop a controller that takes into account the state-dependent delays and compare it with the one presented here. This is going to shed more light on the importance of these delays under various demand variations. Designing controllers for statedependent nonlinear delayed systems is a complex and tedious procedure (see Bekiaris-Liberis and Krstic (2013) for more details). One approach to deal with such systems, is to design a nominal regulator that stabilizes the delayfree system and then apply it to the original delayed system and explore its feasibility and efficiency under different conditions (such designs are presented in Bekiaris-Liberis and Krstic (2013)). This is a modelling simplification that we make for the model-based design of the controller and further investigations are needed in order to assess the theoretical and practical limitations of this assumption. Future research is going to deal with a detailed study of the delayed system and explore more advanced control designs (e.g. feedback law dependent on the delays, predictor feedback control law).

Estimation of $n_{i j}$ states by combining loop detector data and vehicle trajectories (probes), as well as efficient techniques for optimal distribution of the queued vehicles on the controlled links at the boundaries or regions should also be research priorities. Finally, a field implementation of the proposed approach and evaluation in real life conditions could provide further insights about its benefits. Such a case study is under development in the city of Geneva in Switzerland.

\section{Acknowledgements}

This research has been supported by the Swiss National Science Foundation (SNSF) Grant \#200021-146488 and the ERC (European Research Council) Starting Grant "METAFERW: Modelling and controlling traffic congestion and propagation in large-scale urban multimodal networks" (Grant \#338205). The authors are grateful to TSS Company and Dr. Jordi Casas for providing the network of Barcelona. 


\section{References}

Aboudolas, K., Geroliminis, N., 2013. Perimeter and boundary flow control in multi-reservoir heterogeneous networks. Transportation Research Part B $55,265-281$.

Aboudolas, K., Papageorgiou, M., Kouvelas, A., Kosmatopoulos, E., 2010. A rolling-horizon quadratic-programming approach to the signal control problem in large-scale congested urban road networks. Transportation Research Part C 18 (5), 680-694.

Ampountolas, K., Kouvelas, A., 2015. Real-time estimation of critical vehicle accumulation for maximum network throughput. In: American Control Conference. pp. 2057-2062.

Arnott, R., 2013. A bathtub model of downtown traffic congestion. Journal of Urban Economics 76, 110-121.

Bekiaris-Liberis, N., Krstic, M., 2013. Nonlinear control under nonconstant delays. Vol. 25. Siam.

Brown, R. G., Hwang, P. Y., 1997. Introduction to random signals and applied Kalman filtering: with MATLAB exercises and solutions. 3rd edition, New York: Wiley.

Buisson, C., Ladier, C., 2009. Exploring the impact of homogeneity of traffic measurements on the existence of macroscopic fundamental diagrams. Transportation Research Record (2124), 127-136.

Daganzo, C. F., 2007. Urban gridlock: macroscopic modeling and mitigation approaches. Transportation Research Part B 41 (1), 49-62.

Diakaki, C., Dinopoulou, V., Aboudolas, K., Papageorgiou, M., Ben-Shabat, E., Seider, E., Leibov, A., 2003. Extensions and new applications of the traffic-responsive urban control strategy: Coordinated signal control for urban networks. Transportation Research Record 1856 (1), 202-211.

Diakaki, C., Papageorgiou, M., Aboudolas, K., 2002. A multivariable regulator approach to traffic-responsive network-wide signal control. Control Engineering Practice 10 (2), 183-195. 
Gartner, N., 1983. OPAC: A demand-responsive strategy for traffic signal control. Transportation Research Record (906), 75-84.

Gayah, V. V., Daganzo, C. F., 2011a. Clockwise hysteresis loops in the macroscopic fundamental diagram: an effect of network instability. Transportation Research Part B 45 (4), 643-655.

Gayah, V. V., Daganzo, C. F., 2011b. Effects of turning maneuvers and route choice on a simple network. Transportation Research Record (2249), 15-19.

Gayah, V. V., Gao, X. S., Nagle, A. S., 2014. On the impacts of locally adaptive signal control on urban network stability and the macroscopic fundamental diagram. Transportation Research Part B 70, 255-268.

Geroliminis, N., Daganzo, C. F., 2007. Macroscopic modeling of traffic in cities. In: Transportation Research Board 86th Annual Meeting. No. 07-0413.

Geroliminis, N., Daganzo, C. F., 2008. Existence of urban-scale macroscopic fundamental diagrams: Some experimental findings. Transportation Research Part B 42 (9), 759-770.

Geroliminis, N., Haddad, J., Ramezani, M., 2013. Optimal perimeter control for two urban regions with macroscopic fundamental diagrams: A model predictive approach. IEEE Transactions on Intelligent Transportation Systems 14 (1), 348-359.

Geroliminis, N., Sun, J., 2011a. Hysteresis phenomena of a macroscopic fundamental diagram in freeway networks. Transportation Research Part A 45 (9), 966-979.

Geroliminis, N., Sun, J., 2011b. Properties of a well-defined macroscopic fundamental diagram for urban traffic. Transportation Research Part B $45(3), 605-617$.

Godfrey, J. W., 1969. The mechanism of a road network. Traffic Engineering \& Control 11 (7), 323-327.

Haddad, J., 2015. Robust constrained control of uncertain macroscopic fundamental diagram networks. Transportation Research Part C 59, 323-339. 
Haddad, J., Geroliminis, N., 2012. On the stability of traffic perimeter control in two-region urban cities. Transportation Research Part B 46 (9), 1159 1176.

Haddad, J., Mirkin, B., 2016. Adaptive perimeter traffic control of urban road networks based on MFD model with time delays. International Journal of Robust and Nonlinear Control.

Haddad, J., Ramezani, M., Geroliminis, N., 2013. Cooperative traffic control of a mixed network with two urban regions and a freeway. Transportation Research Part B 54, 17-36.

Haddad, J., Shraiber, A., 2014. Robust perimeter control design for an urban region. Transportation Research Part B 68, 315-332.

Ji, Y., Geroliminis, N., 2012. On the spatial partitioning of urban transportation networks. Transportation Research Part B 46 (10), 1639-1656.

Keyvan-Ekbatani, M., Kouvelas, A., Papamichail, I., Papageorgiou, M., 2012. Exploiting the fundamental diagram of urban networks for feedback-based gating. Transportation Research Part B 46 (10), 1393-1403.

Keyvan-Ekbatani, M., Yildirimoglu, M., Geroliminis, N., Papageorgiou, M., 2015. Multiple concentric gating traffic control in large-scale urban networks. IEEE Transactions on Intelligent Transportation Systems 16 (4), 2141-2154.

Knoop, V., Hoogendoorn, S., Van Lint, J., 2012. Routing strategies based on macroscopic fundamental diagram. Transportation Research Record (2315), $1-10$.

Knoop, V. L., Hoogendoorn, S. P., 2014. Network transmission model: a dynamic traffic model at network level. In: Transportation Research Board 93rd Annual Meeting. No. 14-1104.

Kosmatopoulos, E. B., Kouvelas, A., 2009. Large scale nonlinear control system fine-tuning through learning. IEEE Transactions on Neural Networks 20 (6), 1009-1023.

Kouvelas, A., Aboudolas, K., Kosmatopoulos, E. B., Papageorgiou, M., 2011a. Adaptive performance optimization for large-scale traffic control systems. IEEE Transactions on Intelligent Transportation Systems 12 (4), 1434-1445. 
Kouvelas, A., Aboudolas, K., Papageorgiou, M., Kosmatopoulos, E. B., 2011 b. A hybrid strategy for real-time traffic signal control of urban road networks. IEEE Transactions on Intelligent Transportation Systems 12 (3), 884-894.

Kouvelas, A., Lioris, J., Fayazi, S. A., Varaiya, P., 2014. Maximum pressure controller for stabilizing queues in signalized arterial networks. Transportation Research Record (2421), 133-141.

Kouvelas, A., Saeedmanesh, M., Geroliminis, N., 2015. Feedback perimeter control for heterogeneous urban networks using adaptive optimization. In: Proc. 18th IEEE International Conference on Intelligent Transportation Systems. pp. 882-887.

Lamotte, R., Geroliminis, N., 2016. The morning commute in urban areas: Insights from theory and simulation. In: Transportation Research Board 95th Annual Meeting. No. 16-2003.

Leclercq, L., Chiabaut, N., Trinquier, B., 2014. Macroscopic fundamental diagrams: A cross-comparison of estimation methods. Transportation Research Part B 62, 1-12.

Mahmassani, H. S., Saberi, M., Zockaie, A., 2013. Urban network gridlock: Theory, characteristics, and dynamics. Transportation Research Part C 36, 480-497.

Mazloumian, A., Geroliminis, N., Helbing, D., 2010. The spatial variability of vehicle densities as determinant of urban network capacity. Philosophical Transactions of the Royal Society A 368 (1928), 4627-4647.

Mirchandani, P., Head, L., 1998. RHODES - A real-time traffic signal control system: Architecture, algorithms, and analysis. In: TRISTAN III. Vol. 2. San Juan, Puerto Rico.

Muralidharan, A., Pedarsani, R., Varaiya, P., 2015. Analysis of fixed-time control. Transportation Research Part B 73, 81-90.

Ortigosa, J., Menendez, M., Tapia, H., 2014. Study on the number and location of measurement points for an MFD perimeter control scheme: a case study of Zurich. EURO Journal on Transportation and Logistics $3(3-4), 245-266$. 
Papageorgiou, M., Diakaki, C., Dinopoulou, V., Kotsialos, A., Wang, Y., 2003. Review of road traffic control strategies. Proceedings of the IEEE 91 (12), 2043-2067.

Papageorgiou, M., Leibold, M., Buss, M., 2012. Optimierung: statische, dynamische, stochastische Verfahren. 3rd edition, Springer-Verlag, Berlin.

Ramezani, M., Haddad, J., Geroliminis, N., 2015. Dynamics of heterogeneity in urban networks: aggregated traffic modeling and hierarchical control. Transportation Research Part B 74, 1-19.

Saberi, M., Mahmassani, H., 2012. Exploring properties of network-wide flow-density relations in a freeway network. Transportation Research Record (2315), 153-163.

Saeedmanesh, M., Geroliminis, N., 2016. Clustering of heterogeneous networks with directional flows based on "Snake" similarities. Transportation Research Part B 91, 250-269.

Varaiya, P., 2013. Max pressure control of a network of signalized intersections. Transportation Research Part C 36, 177-195.

Yildirimoglu, M., Geroliminis, N., 2014. Approximating dynamic equilibrium conditions with macroscopic fundamental diagrams. Transportation Research Part B 70, 186-200.

Yildirimoglu, M., Ramezani, M., Geroliminis, N., 2015. Equilibrium analysis and route guidance in large-scale networks with MFD dynamics. Transportation Research Part C 59, 404-420. 